\title{
High-velocity resistance training mitigates physiological and functional impairments in middle-aged and older adults with and without mobility-limitation
}

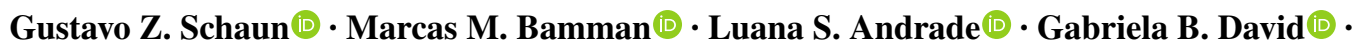 \\ Vitor L. Krüger • Eduardo F. Marins ${ }^{\circledR} \cdot$ Gabriela N. Nunes ${ }^{\circledR}$ - Mariana S. Häfele • Graciele F. Mendes •

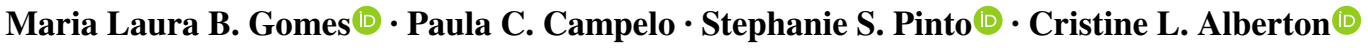

Received: 26 July 2021 / Accepted: 17 January 2022 / Published online: 27 January 2022

(C) The Author(s), under exclusive licence to American Aging Association 2022

\begin{abstract}
The aim of the present study was to compare the neuromuscular, morphological, and functional responses to a high-velocity resistance training (HVRT) program between three cohorts: middle-aged adults (40-55 years, $n=18$ ), healthy older adults ( $>60$ years, $n=18$ ), and mobility-limited older adults $(n=8)$. Participants were tested before and after a 4-week control period and then assigned to a 12-week HVRT intervention. Investigated outcomes included ultrasound-derived muscle thickness and quality, maximal dynamic strength (1RM), maximal voluntary isometric contraction (MVIC), and muscle activation (sEMG), as well as muscle power
\end{abstract}

Supplementary Information The online version contains supplementary material available at https://doi. org/10.1007/s11357-022-00520-8.

G. Z. Schaun $(\bowtie) \cdot$ L. S. Andrade · G. B. David •

V. L. Krüger · E. F. Marins · G. N. Nunes · M. S. Häfele ·

G. F. Mendes · M. L. B. Gomes · P. C. Campelo ·

S. S. Pinto · C. L. Alberton

Neuromuscular Assessment Laboratory, Physical

Education School, Universidade Federal de Pelotas,

Pelotas, RS, Brazil

e-mail: gustavoschaun@hotmail.com

G. Z. Schaun · M. M. Bamman

UAB Center for Exercise Medicine, University of Alabama

at Birmingham, Birmingham, AL, USA

M. M. Bamman

Florida Institute for Human and Machine Cognition,

Pensacola, FL, USA and functional performance. After the intervention, quadriceps muscle thickness, $1 \mathrm{RM}$, and sEMG improved in all three groups (all $p<0.05$ ), whereas muscle quality improved only in middle-aged and older participants $(p \leq 0.001)$, and MVIC only in middle-aged and mobility-limited older adults $(p<0.05)$. With a few exceptions, peak power improved in all groups from 30-90\% 1RM $(p<0.05)$ both when tested relative to pre-training or post-training 1RM workloads (all $p<0.05$ ). Both mobility-limited older adults and older adults improved their short physical performance battery score $(p<0.05)$. Chair stand, stair climb, maximal gait speed, and timed up-andgo performance, on the other hand, improved in all three groups $(p<0.05)$, but no change was observed for habitual gait speed and 6-min walk test performance. Overall, our results demonstrate that a HVRT intervention can build a stronger foundation in middle-aged individuals so that they can better deal with age-related impairments at the same time that it can mitigate already present physiological and functional impairments in older adults with and without mobility-limitation.

Keywords Aged - Exercise - Resistance training · Physical functional performance $\cdot$ Mobility limitation 


\section{Introduction}

The number of middle-aged and older adults in the population is expanding [1,2]. As these individuals age, a series of physiological declines are observed, such as reductions in muscle mass, strength, and power [3]. Recent evidence indicates that the annual reduction rates in power $(\sim 3-6 \%)$ are higher when compared to those of muscle mass and strength $(\sim 1-3 \%)$ [4-8]. This considerable muscle power loss during the aging process is worrisome because power is associated with and is an important predictor of functional performance in elderly people [9-12]. It should be noted, however, that the loss of lower limb muscle mass, strength, and power seems to begin as early as the fourth decade and, therefore, it is important that preventive measures be implemented as soon as possible and not just later in life $[13,14]$.

Progressive resistance training is recommended as a safe and effective way to prevent neuromuscular impairments and to maintain functional performance in individuals over 50 years [15-20]. High-velocity resistance training (HVRT), specifically, seems superior to traditional resistance training for improving muscle power [16, 21] and physical functioning [16] in these individuals, whereas both training modes lead to considerable strength and muscle mass adaptations [21-23]. These results may be achieved using only low to moderate loads, which may be advantageous for naïve exercisers and those with greater limitations, such as mobility-limited older adults. In fact, both middle-aged and older healthy adults [24-28], as well as older adults with limited mobility [29-35], can increase muscle power and improve functional performance after a structured HVRT program.

The majority of previous investigations comparing older adults with and without mobility limitations, as well as middle-aged adults, are observational in nature [9, 36, 37], whereas most experimental trials investigating the effects of HVRT analyzed only one of these groups at a time. Collectively, these investigations suggest that elderly individuals' capacity to respond to the training stimuli remains mostly preserved $[15,16,20]$, but differences in protocols, exercises, training volume, intensity, intervention duration, and outcomes of interest make it difficult to compare adaptations to HVRT in these different groups across separate studies.
It was recently proposed that the mechanisms involved in the loss of muscle power may differ between healthy older adults and those with mobility limitations [7]. After a 3-year follow-up period and similar reductions in lower limb muscle power, only mobility-limited older individuals presented reductions in muscle mass and strength, whereas only the healthy participants showed a reduction in the rate of neuromuscular activation of the agonist's muscles. The same research group had already demonstrated differences in the associations between lower limb muscle power and its determinants (i.e., force and velocity) between middle-aged adults, healthy older adults, and mobility-limited older adults [37]. That is, whereas only force significantly explained the variation in muscle power in the middle-aged participants, both force and velocity explained muscle power in healthy elderly individuals and only velocity in mobility-limited older adults. Collectively, these results suggest that velocity's importance for muscle power production increases with both age and severity of mobility impairment. As such, it is possible that even if these different individuals respond similarly to a HVRT program, the mechanisms driving the responses may differ according to the group being studied.

The magnitude of muscle power gains are similar when HVRT is performed using high or low loads [34, 38, 39]; however, the relationship between muscle power and functional performance can differ according to the relative load in which muscle power is measured and the functional test chosen [40]. That is, whereas power measured at 40 and $70 \%$ of one-repetition maximum (1RM) explained the time required to climb a flight of stairs and to rise from a chair similarly, gait speed was better explained by power measured at the lower intensity. Investigating how HVRT affects muscle power, force, and velocity throughout different percentages of 1RM may, therefore, provide additional information to better understand the adaptations, or lack thereof, in certain functional tests [41].

To the best of our knowledge, no prior trial has compared HVRT adaptations between these different groups of individuals prescribed the same training program and the possible differences in training adaptations. Thus, the aim of the present study was to compare the neuromuscular, morphological, and functional responses to a HVRT program across three 
cohorts: middle-aged adults, healthy older adults, and mobility-limited older adults. Our working hypothesis was that the three groups would improve their neuromuscular and morphological measures, whereas functional capacity would only improve in the older adults' groups and to a higher degree on the mobility-limited individuals. In addition, we hypothesized that improvements in muscle power between the groups would result from distinct contributions from force and velocity. These results have the potential to increase our understanding of cohort-specific HVRT adaptations and to improve HVRT prescription strategies according to the specific needs of each group.

\section{Methods}

\section{Participants}

Middle-aged adults (40-55 years) and older adults (>60 years) with and without mobility limitations were included in the present investigation. The sample size was calculated based on the effect size of previous intervention studies on older adults with mobility limitations or self-reported limitation [e.g., 42, 43, 44], as well as on the effect size of a recent meta-analysis that investigated the effect of HVRT on lower limb muscle power in middle-aged and elderly adults
[20]. Thus, considering $\alpha=0.05,80 \%$ power, and an effect size of 0.22 , a total sample of 54 individuals, 18 per group, was estimated. The study was advertised through newspapers and social media. Initial contact with potential participants was made through telephone pre-screens, during which the inclusion and exclusion criteria (Table 1) were reviewed using a standardized form; those deemed eligible and that remained interested were then invited for an in-person interview at the university. Individuals over 60 years of age were also asked about possible mobility limitations [i.e., self-reported difficulty in at least one of the following tasks: walking $400 \mathrm{~m}$, climbing stairs, lifting and carrying groceries, getting out of the bathtub or shopping, 45].

At the university, volunteers answered a health questionnaire to verify their eligibility and then signed an informed consent form to participate in the study, which was approved by the Federal University of Pelotas Ethics Review Board (CAAE: $13,459,719.0 .0000 .5313)$ in accordance with the Declaration of Helsinki. During this visit, older participants also performed the SPPB, and those with a score of $\leq 9$ points were classified as being mobility-limited for the purpose of the present study [46, 47]. To minimize the potential impact of differential levels of physical activity, we restricted recruitment to untrained subjects (i.e., no recent

Table 1 Eligibility criteria

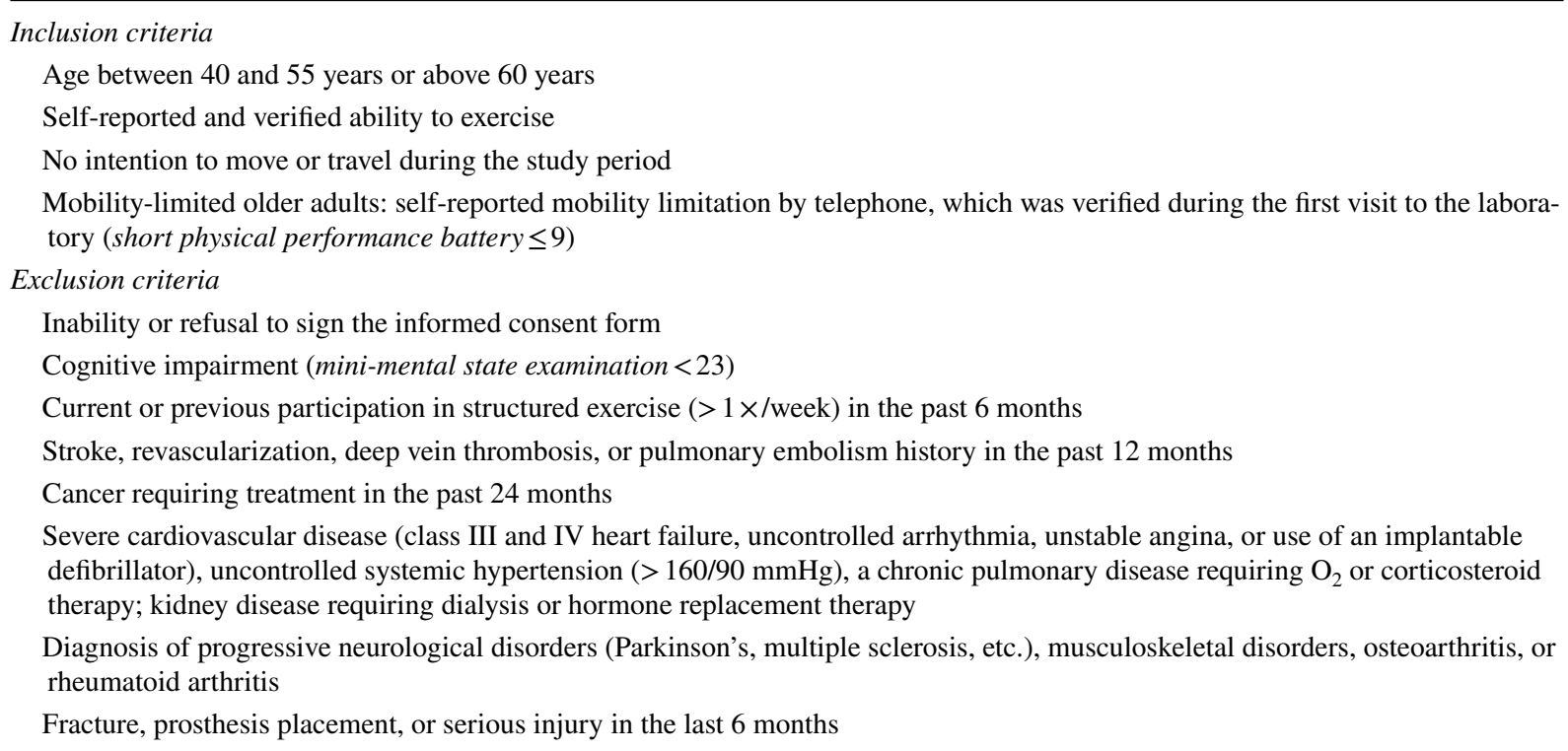


training history, as highlighted in Table 1). As part of the screening process, we also captured physical activity patterns via questionnaires. There were no notable differences in the weekly levels of selfreported physical activity among middle-aged, older adults, and mobility-limited older adults $(p>0.05)$. All subjects reported levels of activity well below the Department of Health and Human Services 2018 physical activity guidelines (i.e., were physically inactive).

\section{Experimental procedures}

The experimental design of the study is shown in Fig. 1. All participants attended two familiarization sessions. During these sessions, they were introduced to the exercises and tests that they would perform with an emphasis on technique and movement velocity. Following familiarization and prior to the intervention, subjects were tested before (baseline) and after (pre-intervention) a 4-week control washin period. In each of these time points, participants completed two days of testing. On the first day, knee extensors ultrasound images were taken followed by knee extension (KE) maximal voluntary isometric contractions, KE 1RM, and power tests, whereas on the second-day leg press (LP) 1RM and power tests were followed by a battery of functional tests. No exercise was scheduled during the control period, and participants were advised to maintain their normal routine throughout it.

Participants were then assigned to a 12-week HVRT program twice per week, and all outcomes were reassessed in three separate visits at least $72 \mathrm{~h}$ after the last training session (post-intervention). The first two sessions were similar to those at the baseline and pre-intervention time points, whereas, in the third session, participants repeated both $\mathrm{KE}$ and LP power tests in a counterbalanced order using the 1RM load corresponding to the baseline time point. All post-intervention tests were completed within two weeks. Throughout the study, both the time $( \pm 2 \mathrm{~h})$ and order of the tests, as well as outcome assessors were kept constant, and the participants were instructed to avoid the consumption of caffeine/alcohol and the practice of vigorous physical activities in the $24 \mathrm{~h}$ preceding them.
Measurements

\section{Ultrasound measures}

In the first testing session, subjects reported to the lab and rested for $5 \mathrm{~min}$ in the supine position [48]. Five images from the rectus femoris (RF), vastus intermedius (VI), vastus lateralis (VL), and vastus medialis (VM) muscles of the right limb were then obtained by B-mode ultrasound (Tosbee/SSA-240a, Toshiba ${ }^{\circledR}$, Japan) using a $7.5 \mathrm{MHz}$ linear-array probe positioned perpendicularly to the muscle of interest. RF, VI, and VL images were obtained at $50 \%$ of the distance between the lateral condyle and the greater trochanter of the femur, whereas VM images were obtained at $20 \%$ of the same distance $[49,50]$. Images were analyzed using the ImageJ software v1.52a (National Institutes of Health, Bethesda, USA). RF, VL, and VM muscle thicknesses were defined as the distance between the superficial and deep muscle aponeurosis, whereas VI thickness corresponded to the distance between the superficial aponeurosis and the hyperechoic interface of the femur. Muscle quality, on the other hand, was determined based on the muscles' echo intensity values using a grayscale analysis function on the ImageJ software, and expressed in arbitrary units between 0 (black, higher quality) and 255 (white, lower quality). For this purpose, in each muscle image a region of interest was selected including as much muscle area as possible while avoiding other tissues (see Supplementary Fig. 1 for a representative ultrasound image). Muscle thickness and echo intensity values are presented for RF, VI, VL, and VM individually as the average value between all its five images and also for the quadriceps femoris as the sum (muscle thickness) and average (echo intensity) of the individual muscle values, respectively. Probe positions were carefully mapped to ensure an identical positioning between baseline, pre-intervention, and post-intervention time points [51, 52].

\section{Maximal isometric contraction}

After the ultrasound measures were taken, participants undertook a maximal voluntary unilateral knee extension isometric contraction (MVIC) test. They were positioned on the knee extension machine (NEWFIT ${ }^{\circledR}$, Cascavel, Brazil) at $90^{\circ}$ of hip and knee flexion and were instructed to exert maximal force as 


\begin{tabular}{|c|c|l|}
\hline \multirow{2}{*}{ Phase } & Day & \multicolumn{1}{c|}{ Tests } \\
\hline \multirow{2}{*}{ Familiarization } & 1 & Familiarization with exercises \\
\cline { 2 - 3 } & 2 & Familiarization with exercises; 1RM estimation \\
\hline
\end{tabular}

\begin{tabular}{|c|c|c|l|}
\hline \multicolumn{4}{|c|}{ Pre-intervention } \\
\hline Phase & Week & Day & Tests \\
\hline \multirow{2}{*}{ Baseline } & & 1 & Ultrasound; MVIC + sEMG; KE 1RM + Power (30-90\% 1RM) \\
\cline { 3 - 5 } & & 2 & LP 1RM + Power (30-90\% 1RM); Functional measures \\
\hline
\end{tabular}

\begin{tabular}{|c|c|c|l|}
\hline Phase & Week & Day & Tests \\
\hline \multirow{2}{*}{ Pre } & & 1 & Ultrasound; MVIC + EMG; KE 1RM + Power (30-90\% 1RM) \\
\cline { 3 - 5 } & 0 & 2 & LP 1RM + Power (30-90\% 1RM); Functional measures \\
\hline
\end{tabular}

\begin{tabular}{|c|c|c|c|c|c|c|}
\hline \multicolumn{7}{|c|}{ Intervention (12 weeks) } \\
\hline Exercises & Week & Day & Volume (sets $x$ reps) \& Load & Week & Day & Volume (sets $x$ reps) \& Load \\
\hline \multirow{12}{*}{ 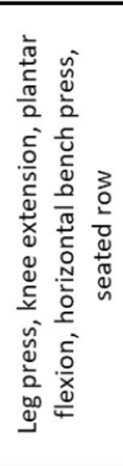 } & \multirow{2}{*}{1} & 1 & $1 \times 8-10 @ 40 \%$ 1RM* & \multirow{2}{*}{7} & 1 & $3 \times 8-10 @ 40 \% 1 \mathrm{RM}$ \\
\hline & & 2 & $1 \times 8-10 @ 60 \% 1 R M$ & & 2 & $3 \times 8-10 @ 60 \% 1 \mathrm{RM}$ \\
\hline & \multirow{2}{*}{2} & 1 & 2x8-10@40\% 1RM+ & \multirow{2}{*}{8} & 1 & $3 \times 8-10 @ 40 \% 1 \mathrm{RM}$ \\
\hline & & 2 & $2 \times 8-10 @ 60 \% 1 \mathrm{RM}$ & & 2 & $3 \times 8-10 @ 60 \% 1 \mathrm{RM}$ \\
\hline & \multirow{2}{*}{3} & 1 & $2 \times 8-10 @ 40 \% 1 R M$ & \multirow{2}{*}{9} & 1 & $3 \times 8-10 @ 40 \% 1 \mathrm{RM}$ \\
\hline & & 2 & $2 \times 8-10 @ 60 \% 1 R M$ & & 2 & $3 \times 8-10 @ 60 \% 1 \mathrm{RM}$ \\
\hline & \multirow{2}{*}{4} & 1 & $2 \times 8-10 @ 40 \% 1 R M$ & \multirow{2}{*}{10} & 1 & $3 \times 8-10 @ 40 \% 1 \mathrm{RM}$ \\
\hline & & 2 & $2 \times 8-10 @ 60 \% 1 R M$ & & 2 & $3 \times 8-10 @ 60 \% 1 \mathrm{RM}$ \\
\hline & \multirow{2}{*}{5} & 1 & $2 \times 8-10 @ 40 \% 1 R M$ & \multirow{2}{*}{11} & 1 & $3 \times 8-10 @ 40 \% 1 \mathrm{RM}$ \\
\hline & & 2 & $2 \times 8-10 @ 60 \% 1 R M$ & & 2 & $3 \times 8-10 @ 60 \% 1 \mathrm{RM}$ \\
\hline & \multirow{2}{*}{$6^{\ddagger}$} & 1 & $2 \times 8-10 @ 40 \% 1 R M$ & \multirow{2}{*}{12} & 1 & $3 \times 8-10 @ 40 \% 1 \mathrm{RM}$ \\
\hline & & 2 & 2 x 8-10@60\% 1RM & & 2 & 3 x 8-10@60\% 1RM \\
\hline
\end{tabular}

\begin{tabular}{|c|c|c|l|}
\hline \multirow{2}{*}{ Phase } & Week & Day & \multicolumn{1}{|c|}{ Post-intervention } \\
\hline \multirow{2}{*}{ Post } & \multirow{2}{*}{13} & 1 & Ultrasound; MVIC + EMG; KE 1RM + Power (30-90\% post-training 1RM) \\
\cline { 3 - 5 } & & 2 & LP 1RM + Power (30-90\% post-training 1RM); Functional measures \\
\cline { 2 - 5 } & 14 & 3 & KE and LP Power (30-90\% pre-training 1RM) \\
\hline
\end{tabular}

Fig. 1 Experimental design of the study. 1RM, one-repetition maximum test; MVIC, maximal voluntary isometric contraction; sEMG, surface electromyography; KE, knee extension; LP, leg press. *for the exercises beyond KE and LP,

fast as possible while receiving strong verbal encouragement. Each participant had three attempts lasting $\sim \mathrm{s}$, and 2 min were allowed between attempts. initial training loads were determined based on predicted $\% 1$ RM (BRZYCKI, 1993); $\dagger 2$ min between sets; $\ddagger$ loads were increased by $5 \%$ after week 6

The maximal isometric force was measured using a load cell (Miotec®, Porto Alegre, Brazil) with 200 kgf capacity. 


\section{Surface EMG measures}

Neuromuscular activation of the RF and VL muscles and biceps femoris (BF) antagonist coactivation were measured during the MVIC test using surface electromyography (sEMG; Miotool400, Miotec $®$, Porto Alegre, Brazil). Briefly, $\mathrm{Ag} / \mathrm{AgCl}$ electrodes (Meditrace ${ }^{\circledR}$, Mansfield, Canada) were positioned in bipolar configuration (30 $\mathrm{mm}$ interelectrode distance) on the muscle belly of each muscle in participants' right limb following established recommendations [53], and a reference electrode was also placed on the tibial tuberosity. Electrode positions were carefully mapped to ensure an identical positioning between baseline, pre-intervention, and post-intervention time points $[51,52]$.

Both force and sEMG signals were collected using the MiotecSuite software (Miotec $\AA$, Porto Alegre, Brazil) and stored for subsequent analysis on LabView 2019 (National Instruments, Austin, United States). Force signals were filtered using a low-pass fifth-order Butterworth digital filter at a cutoff frequency of $8 \mathrm{~Hz}$, and sEMG signals were band-pass filtered by a fifth-order Butterworth digital filter at a frequency range set between 20 and $500 \mathrm{~Hz}$. The maximal isometric force (kgf) and the root mean square value of the sEMG signal $(\mu \mathrm{V})$ were then determined in the highest 1 -s epoch of the force-time signal.

\section{Maximal dynamic strength}

Maximal dynamic strength was measured via bilateral KE and LP 1RM tests (New Fitness ${ }^{\circledR}$, São Paulo, Brazil), as shown in Fig. 1. Participants first performed a 10 repetitions warm-up set at a load equivalent to $50 \%$ of their estimated 1RM, which was determined during familiarization, and the resistance was increased until only one repetition could be performed using appropriate technique and range of motion. After each attempt, resistance was adjusted based on the Lombardi scale (1989). 1RM load (in $\mathrm{kg}$ ) was determined in no more than five attempts separated by $3 \mathrm{~min}$ intervals. To ensure a reliable measure, both KE and LP range of motion were registered during the baseline sessions using a custombuilt device similar to the one applied by Radaelli et al. [28] and replicated during both pre- and postintervention time points.

\section{Muscle power}

Following completion of the 1RM tests, participants rested for 20-30 min and then performed KE and LP power testing by completing one repetition with each load corresponding to $30,40,50,60,70,80$, and $90 \%$ $1 \mathrm{RM}$ with $1 \mathrm{~min}$ intervals between each set [10, 45, 54]. Participants were instructed to perform the concentric phase of each repetition as fast as possible and the eccentric phase in $2 \mathrm{~s}$ [45]. For each repetition, the peak velocity, force, and power were determined during the concentric portion of the exercises using a linear position transducer (Chronojump BoscoSystem ${ }^{\circledR}$, Barcelona, Spain) attached to the weight stack on the KE machine and perpendicular to the motion trajectory on the LP machine sled to ensure a direct vertical displacement in both exercises. [30]. Similar to the 1RM test, both KE and LP range of motion were registered during the baseline sessions using the same custom-built device and were replicated during both pre- and post-intervention time points. In addition, during post-intervention assessments, power tests were performed both at $30-90 \%$ of the postand pre-intervention 1RM loads in separate visits, as shown in Fig. 1 [55], to assess changes in both relative and absolute power.

\section{Functional performance}

Short physical performance battery The SPPB is a validated, widely employed test to assess functional capacity due to its strong association with disability and mobility limitation, and because it is also a predictor of institutionalization and mortality [46, 56]. The battery is based on three different tasks: static balance, gait speed, and chair stand. For the static balance test, participants were instructed to stand for $10 \mathrm{~s}$ in three different positions, feed side-by-side, semi-tandem stance, and tandem stance. Gait speed was measured based on the time required for the participants to walk a 4-m distance at their habitual gait speed, and chair rise performance was measured through the time required for participants to rise from a chair $(0.43 \mathrm{~m}$ high $)$ and return to the seated position five times as fast as possible. Based on objective criteria, each task receives a score between 0 and 4 points, and the sum of the individual scores determines the final SPPB score ranging from 0 to 12 (lower values indicate worse functional performance). 
Gait speed Although gait speed is part of the SPPB test, it is also an individual predictor of disability and all-cause mortality [57-59] and, therefore, it was also measured separately in the present study. Participants were instructed to walk a $10-\mathrm{m}$ distance at their habitual and maximal gait speeds $[60,61]$ in separated trials. To avoid acceleration and deceleration interference, a 1-m distance both before and after the $10-\mathrm{m}$ track was provided. The time required to cover the distance was measured using a stopwatch, and the corresponding gait speed was determined in $\mathrm{m} \cdot \mathrm{s}^{-1}$. Each participant had two attempts per speed with 1 min interval between them, and the best result was used for analysis. The use of a long-distance in comparison to the SPPB (10 vs. $4 \mathrm{~m}$ ), as well as additional testing maximal gait speed, aims to provide a greater sensitivity to discriminate between the three groups investigated [60].

Chair stand The chair stand test is also part of the SPPB battery, however, we chose to measure it separately to better discriminate the groups investigated and also to eliminate possible ceiling effects [as previously suggested by 62]. Participants began seated on a chair ( $0.43 \mathrm{~m}$ height) with both arms crossed over their chest and feet shoulder-width apart and were instructed to rise up as many times as possible in $30 \mathrm{~s}$. Only one attempt of this test was performed, and the number of repetitions was used for the analysis. The major difference between this specific test and the chair stand in the SPPB is its focus on maximal repetition number in a standardized amount of time, as opposed to the SPPB, which defines a standardized number of repetitions with time allowed to vary as the dependent variable. Additionally, this $30 \mathrm{~s}$ test enables some assessment of short-term fatigue.

Stair climb Participants were instructed to climb a flight of stairs (10 steps, $17 \mathrm{~cm}$ each) as fast as possible, without using the handrails. The time required to complete the task was measured using a stopwatch from the moment the participants put their foot on the first step to when both feet were on the last step. Each participant had two attempts with 1 min interval between them, and the best time was used for analysis.

Timed up and go Agility and dynamic balance were assessed using the timed up and go (TUG) test.
The test began with participants seated on a chair, and a cone positioned $3 \mathrm{~m}$ away in front of them [63]. At the investigator's command, participants rose from the chair, walked as fast as possible without running around the cone, and returned to the initial seated position. The time required to perform the task was measured using a stopwatch, and the lower value between two attempts was used in the analysis.

6-min walk test The test was performed on a 30-m flat surface in which participants were instructed to walk back and forth as many times as they could for $6 \mathrm{~min}$. Incentives were provided every min (e.g., "You are doing well, there is X min left"). Participants performed the test once and the total distance covered was used in the analysis.

\section{Training program}

After baseline testing, participants underwent a 12-week HVRT program, which is shown in detail in Fig. 1. Participants exercised twice per week on non-consecutive days, and all exercise sessions were supervised by trained instructors (2:1 ratio). After a 10-min warm-up containing general and specific warm-up activities with submaximal loads (i.e., horizontal bike and leg press or knee extension), participants performed 1-3 sets of 8-10 repetitions of 5 different exercises (see Fig. 1 for volume progression). The exercises were leg press, bilateral knee extension, seated plantar flexion, horizontal chest press, and seated row. All individuals were instructed to perform the concentric phase of movements as fast as possible and the eccentric phase in $2 \mathrm{~s}$ while maintaining exercise range of motion.

Exercise intensity was prescribed based on the $1 \mathrm{RM}$ percentage measured during the leg press and knee extension exercises, and on the estimated 1RM percentage for the remaining exercises [64], which was determined during the familiarization session [as performed in 65]. Specifically, the first weekly session was performed at $40 \% 1 \mathrm{RM}$ and the second session at $60 \% 1 \mathrm{RM}$. In addition, the load for each exercise was increased by $5 \%$ after the sixth week of training to account for strength adaptations. 
Statistical analysis

Data are reported as mean \pm standard deviation (SD) unless stated otherwise. Training-related adaptations in the outcomes between pre- and post-training time points were tested using generalized estimating equation models (GEE), and group main effects were analyzed using Bonferroni's post hoc. To confirm the efficacy of the control wash-in period, GEE and Bonferroni's post hoc were also used to compare the outcomes between the control and baseline time points. In the case of a significant interaction, within-group and between-group effects were further tested using Bonferroni's post hoc. In addition, Cohen's d effect sizes were determined and classified as small $(\geq 0.2)$, moderate $(\geq 0.5)$, large $(\geq 0.8)$, and very large $(\geq 1.3)$, as suggested by Sullivan and Feinn (2012). Finally, to gain a better insight on HVRT regenerative capacity, outcomes from the mobility-limited older adults' group that were found to be significantly increased after the intervention were compared to the pre-training values of the older adults group using independent-samples $t$-tests to verify if the training effect was sufficient to bring about a more functional phenotype. The same was also performed for comparing older and middle-aged adults. All analyses were performed using SPSS (v. 25.0., IBM, USA) with a significance level set at $\alpha=0.05$.

\section{Results}

The study workflow is shown in Fig. 2, and the characteristics of the participants included in the analysis are presented in Table 2. Initial contact was made through telephone pre-screens with 180 individuals, 58 of which were deemed as potentially eligible and invited for an in-person interview. Of these, nine were excluded for not meeting the inclusion criteria, and four declined to participate. Thus, forty-five individuals, including 19 middle-aged adults (40-55 years), 18 older adults ( $>60$ years), and 8 mobility-limited older adults ( $>60$ years), volunteered to participate and were included in the study. Although a second recruitment wave was scheduled to achieve the estimated sample size for the mobility-limited older adults' group, the COVID-19 pandemic and the restrictions imposed by it made it impractical/unfeasible to fulfill this goal.
Except for two participants in the middle-aged group, there were no other dropouts throughout the intervention. Of these, one woman was transferred to another city prior to baseline testing, and one had a back injury not related to the intervention at the second week of training. Thus, 43 participants completed the training program, and 44 with pre-intervention data were included in the analyses. Training compliance, i.e., the degree to which participants adhered to an assigned treatment protocol, was high and corresponded to $90.7 \pm 7.3 \%, 94.4 \pm 5.5 \%$, and $90.6 \pm 10.1 \%$ for middle-aged, older, and mobilitylimited older adults, respectively. More specifically, participants attended $952(92.2 \pm 7.3 \%)$ of the 1032 planned training sessions, of which 950/952 (99.8\%) were completed. Finally, twenty-five adverse events from thirteen participants (4 middle-aged, 4 older adults, and 5 mobility-limited older adults) were registered throughout the intervention. The majority were classified as mild $(n=14)$ or moderate $(n=7)$ and were mostly related to musculoskeletal conditions, such as muscle soreness. In addition, only 7 events were considered definitely related to the intervention, of which 4 resulted in acute modifications to the training session (the reader is referred to Supplementary Chart 1 for a detailed list of adverse events).

\section{Control period}

With the exception of LP and KE 1RM $(p=0.007$ and $p=0.001$, respectively), LP peak power at $50 \%$ $(p=0.025)$ and $70 \%(p=0.021)$, KE peak power at $30 \%(p=0.019)$, quadriceps muscle thickness ( $p=0.005$, older adults' group only), and 30STS performance $(p=0.003)$, no other differences were observed between baseline and pre-intervention results (all $>0.05$ ). In addition, despite being statistically different, effect sizes for the aforementioned outcomes were found to be mostly trivial or small. Readers are referred to Supplementary Table 1 for a detailed description of the control period results.

\section{Ultrasound measures}

Quadriceps muscle thickness results are presented in Fig. 3A, whereas individual muscle results are available in Supplementary Fig. 2. Quadriceps muscle thickness increased in all three groups $(p<0.001)$ after training and a group main effect followed by 


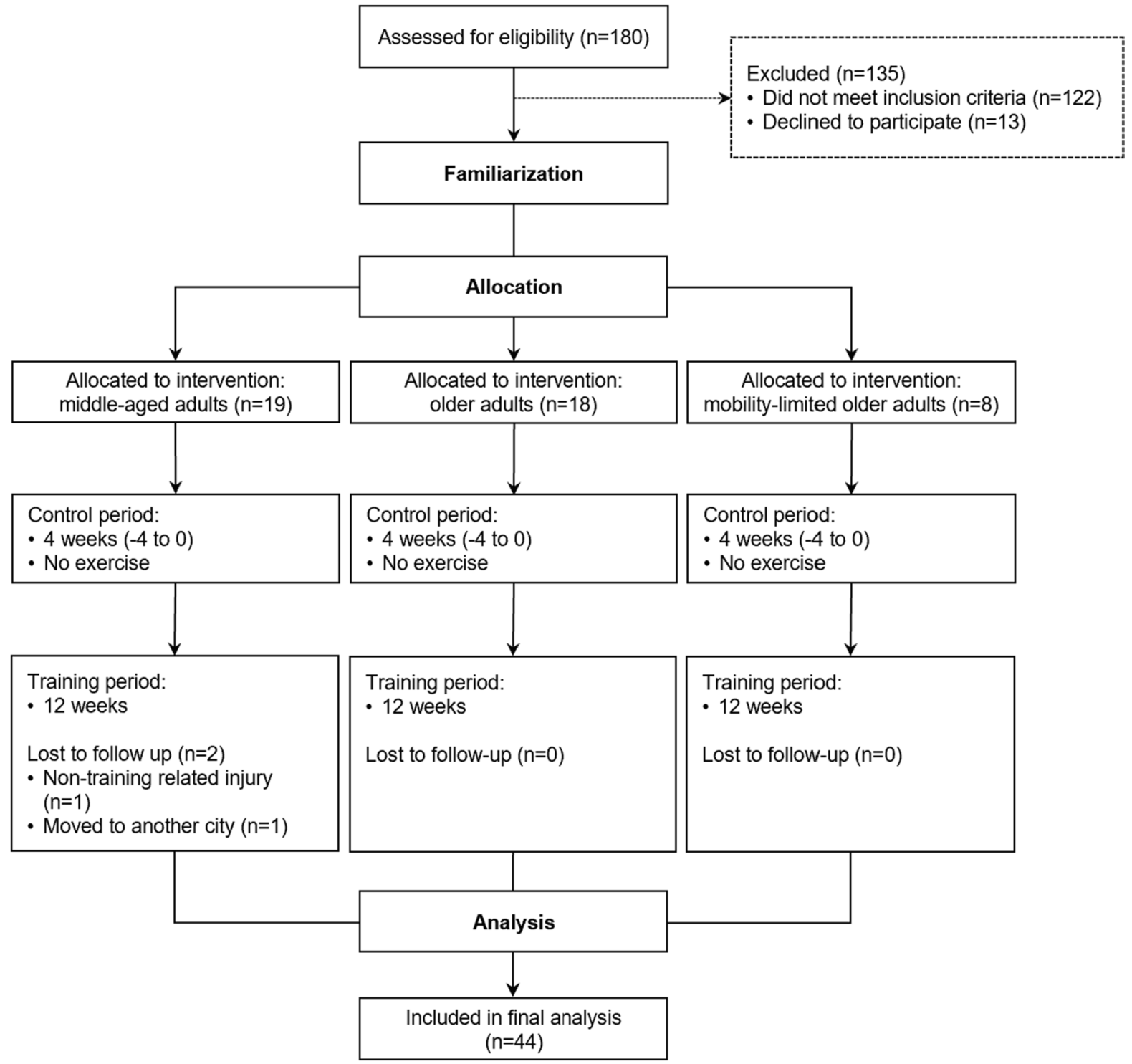

Fig. 2 Study workflow

post hoc analyses showed that middle-aged adults had greater muscle thickness compared to both older adults $(p=0.027)$ and mobility-limited older adults $(p<0.001)$. No difference was found between the two older groups $(p=0.100)$.

Quadriceps muscle quality results are presented in Fig. 3B, whereas individual muscle results are available in Supplementary Fig. 3. When pooled together as a single quadriceps measure, an improvement in muscle quality, as indicated by a reduction in echo intensity, was only found in the middle-aged $(p<0.001)$ and older adults $(p=0.001)$, whereas mobility-limited older adults' quadriceps muscle quality remained unchanged $(p=0.653)$. Moreover, although no difference was observed between older adults and mobility-limited older adults before training $(p=0.171)$, after the intervention the former had a significantly greater muscle quality than the latter $(p=0.001)$, both of which had lesser muscle quality compared to middle-aged participants pre- $(p=0.027$ and $p<0.001$, respectively) and post-training ( $p=0.001$ and $p<0.001$, respectively). 
Table 2 Characteristics of the included participants at baseline

$S B P$, systolic blood pressure; $D B P$, diastolic blood pressure; $S P P B$, short physical performance battery score at baseline

Fig. 3 Quadriceps femoris muscle thickness (A) and echo intensity (B) responses to 12 weeks of high-velocity resistance training in middle-aged (MID), older (OLD) and mobility-limited older (LIM) participants. $*=$ post different than pre (all $p<0.05$ ); $\dagger=$ LIM different than MID (all $p<0.05$ ); $\neq=$ LIM different than OLD (all $p<0.05$ ); $\S=$ OLD different than MID (all $p<0.05$ ). Values are $($ mean $\pm \mathrm{SD})$

\begin{tabular}{llll}
\hline & MID $(n=18)$ & OLD $(n=18)$ & LIM $(n=8)$ \\
\hline Age (years) & Mean \pm SD & Mean \pm SD & Mean \pm SD \\
Height (m) & $48.9 \pm 14.2$ & $68.9 \pm 6.5$ & $77.3 \pm 8.2$ \\
Body mass (kg) & $1.67 \pm 0.09$ & $1.66 \pm 0.09$ & $1.64 \pm 0.07$ \\
Body mass index & $73.3 \pm 13.1$ & $74.5 \pm 14.7$ & $70.2 \pm 12.8$ \\
Waist circumference (cm) & $26.2 \pm 3.1$ & $26.9 \pm 4.3$ & $25.9 \pm 2.6$ \\
SBP (mmHg) & $86.8 \pm 9.9$ & $93.7 \pm 12.8$ & $91.2 \pm 9.6$ \\
DBP (mmHg) & $120.0 \pm 14.2$ & $126.0 \pm 17.3$ & $128.2 \pm 20.1$ \\
SPPB (score) & $76.3 \pm 9.8$ & $71.4 \pm 8.1$ & $67.0 \pm 8.8$ \\
Sex & $11.8 \pm 0.5$ & $11.5 \pm 0.7$ & $8.1 \pm 1.0$ \\
\hline
\end{tabular}

A Quadriceps Femoris पMID DOLD पLIM
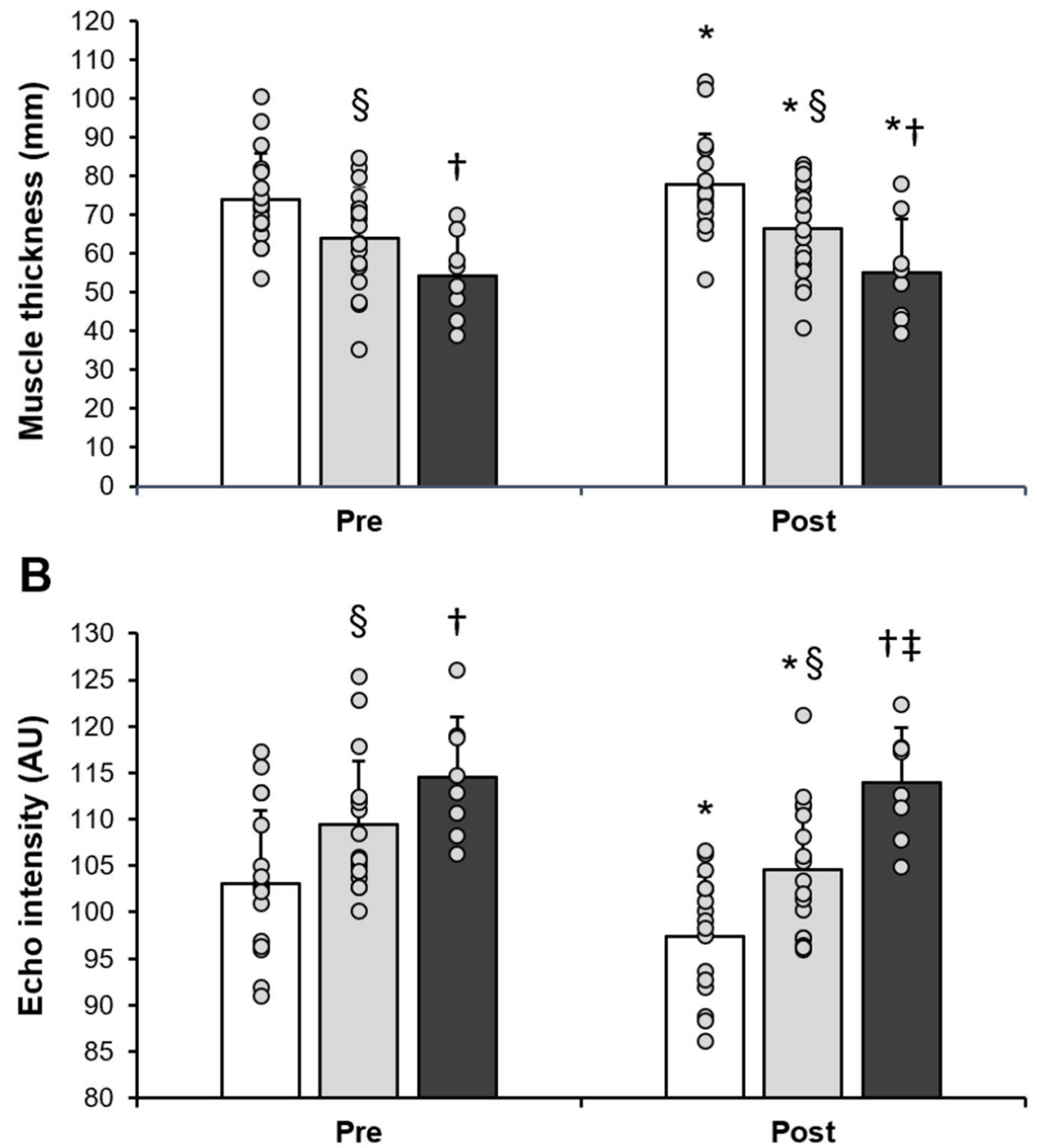
Table 3 Maximal isometric strength and electromyographic responses to 12 weeks of high-velocity resistance training

\begin{tabular}{|c|c|c|c|c|c|c|}
\hline & \multicolumn{2}{|c|}{ Middle-aged adults $(n=18)$} & \multicolumn{2}{|c|}{ Older adults $(n=18)$} & \multicolumn{2}{|c|}{$\begin{array}{l}\text { Mobility-limited older adults } \\
(n=8)\end{array}$} \\
\hline & Pre & Post & Pre & Post & Pre & Post \\
\hline MVIC (kgf) & $33.9 \pm 13.0$ & $37.4 \pm 17.2 *$ & $26.9 \pm 9.8$ & $27.8 \pm 8.7$ & $19.9 \pm 6.5^{\dagger}$ & $22.8 \pm 7.5 * \dagger$ \\
\hline VL sEMG $(\mu \mathrm{V})$ & $384.0 \pm 290.6$ & $414.2 \pm 299.8 *$ & $284.8 \pm 102.4$ & $302.8 \pm 131.8^{*}$ & $209.99 \pm 67.9$ & $229.2 \pm 87.8^{* \dagger}$ \\
\hline RF sEMG $(\mu \mathrm{V})$ & $283.1 \pm 231.0$ & $343.4 \pm 349.1 *$ & $219.4 \pm 82.0$ & $231.3 \pm 101.1^{*}$ & $180.9 \pm 84.4$ & $190.6 \pm 122.1 *$ \\
\hline BF sEMG $(\mu \mathrm{V})$ & $74.6 \pm 38.5$ & $78.8 \pm 45.6$ & $54 \pm 17.7$ & $66.8 \pm 27.9$ & $43.8 \pm 13.4^{\dagger \neq}$ & $42 \pm 18.7^{\dagger \neq}$ \\
\hline
\end{tabular}

$M V I C$, maximal knee extension voluntary isometric contraction; $V L$, vastus lateralis; $R F$, rectus femoris; $B F$, biceps femoris; $s E M G$, surface electromyography (root mean square). $*=$ post greater than pre (all $p<0.05) ; \dagger=$ LIM lower than MID $(p<0.05) ; \ddagger=$ LIM lower than OLD $(p<0.05)$. Values are $($ mean $\pm \mathrm{SD})$

\section{Maximal isometric contraction}

MVIC and sEMG data are shown in Table 3. Both middle-aged adults and mobility-limited older adults improved their maximal isometric strength after the intervention ( $p=0.007$ and $p<0.001$, respectively), but no difference was found in the older adults' group $(p=0.212)$. For the sEMG data, however, both VL and RF RMS sEMG increased in all three groups post-training ( $p=0.14$ and $p=0.032)$, whereas no effect was observed in BF sEMG $(p=0.081)$. Further, the mobility-limited older adults' group was found to have a lower MVIC strength than middle-aged (pre: $p<0.001$; post: $p=0.005$ ), but not older adults (pre: $p=0.085$; post: $p=0.379$ ). VL sEMG also differed between middle-aged and mobility-limited older adults $(p=0.032)$, but RF sEMG was not different between any of the groups $(p>0.05)$. BF sEMG, on the other hand, was lower in the mobility-limited older adults group compared to both middle-aged $(p=0.003)$ and older adults $(p=0.044)$.

\section{Maximal dynamic strength}

Leg press and knee extension 1RM data are presented in Fig. 4. In both exercises, 1RM improved after the intervention in all groups (all $p<0.001$ ). For LP, both pre- and post-intervention mobility-limited older adults had lower 1RM performance when compared to both middle-aged $(p<0.001)$ and older adults $(p=0.032)$, whereas no differences were observed between the other two groups ( $p=0.094)$. Conversely, although the mobility-limited older adults group had a lower KE 1RM than middle-aged $(p<0.001)$ and older adults $(p=0.050)$ pre-intervention, they differed only from the middle-aged group $(p<0.001)$ post-intervention.

Muscle power

For brevity, only peak power results are presented herein. For a comprehensive presentation of KE and LP peak velocity and force results assessed using both pre- and post-training 1RM loads, readers are referred to Supplementary Figs. 4-7.

When power was assessed at intensities relative to the post-intervention 1RM load (Fig. 5A-C), an increase in peak power output was observed for all groups from 40 to $80 \%$ 1RM $(p<0.05)$ in the $\mathrm{KE}$ exercise, but only middle-aged and older participants increased peak power at $30 \%(p<0.05)$. No improvements were found at 90\% 1RM $(p>0.05)$. Mobility-limited older adults had a lower peak power output compared to the other two groups, both preand post-training at all loads (i.e.. 30-90\% 1RM, $\mathrm{p}<0.05$ ), whereas older adults had lower power output than middle-aged participants at $30 \%$ and from 50 to $80 \% 1 \mathrm{RM}(p<0.05)$. An interaction at $40 \%$ $1 \mathrm{RM}$ revealed that although there was no difference between middle-aged and older adults at pre-training $(p>0.05)$, the former had a higher power output at post $(p<0.05)$. No between-group differences were found at $90 \% 1 \mathrm{RM}(p>0.05)$. In the LP exercise (Fig. 5D-F), peak power increased in all groups from 30-90\% 1RM $(p<0.05)$ and, similar to the KE exercise, mobility-limited older adults had a lower power output than middle-aged adults and older adults in all loads both pre- and post-training $(p<0.05)$. No differences were found between middle-aged and older adults $(p>0.05)$. 
Fig. 4 Leg press (A) and Knee extension (B) maximal dynamic strength (1RM) response to 12 weeks of high-velocity resistance training in middle-aged (MID), older (OLD) and mobility-limited older (LIM) participants. $*$ = post greater than pre (all $p<0.05) ; \dagger=$ LIM lower than MID $(p<0.001)$ and OLD $(p=0.032) ; \ddagger=$ LIM lower than MID $(p<0.001)$. Values are $($ mean $\pm \mathrm{SD})$
A

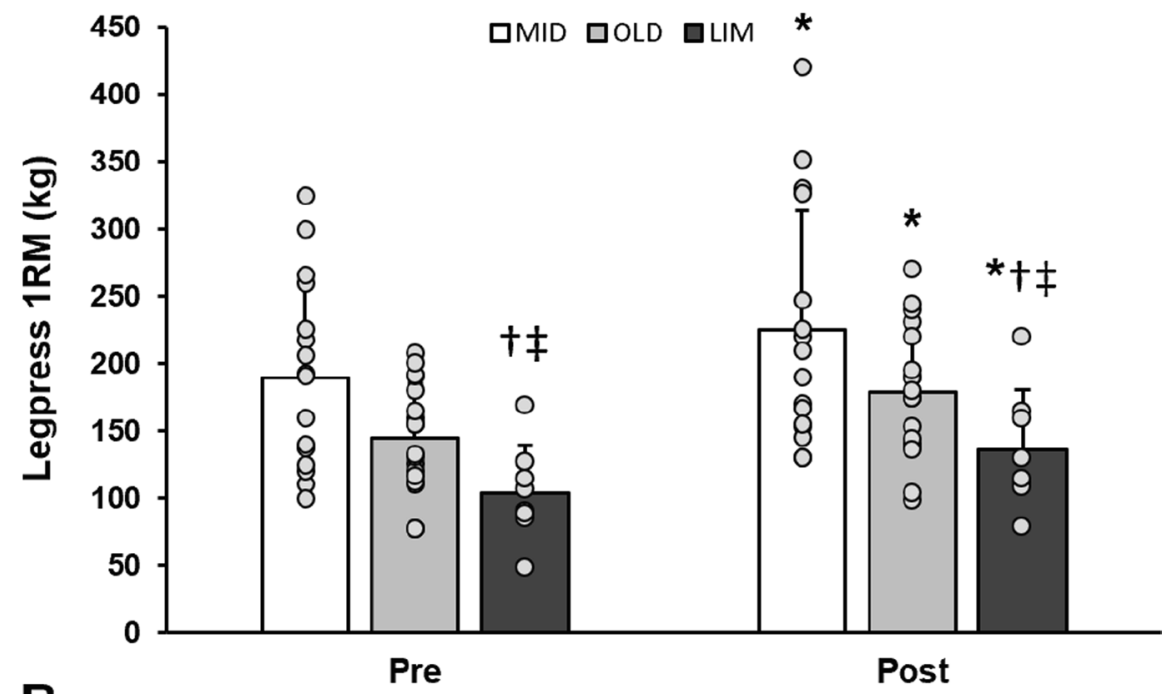

B

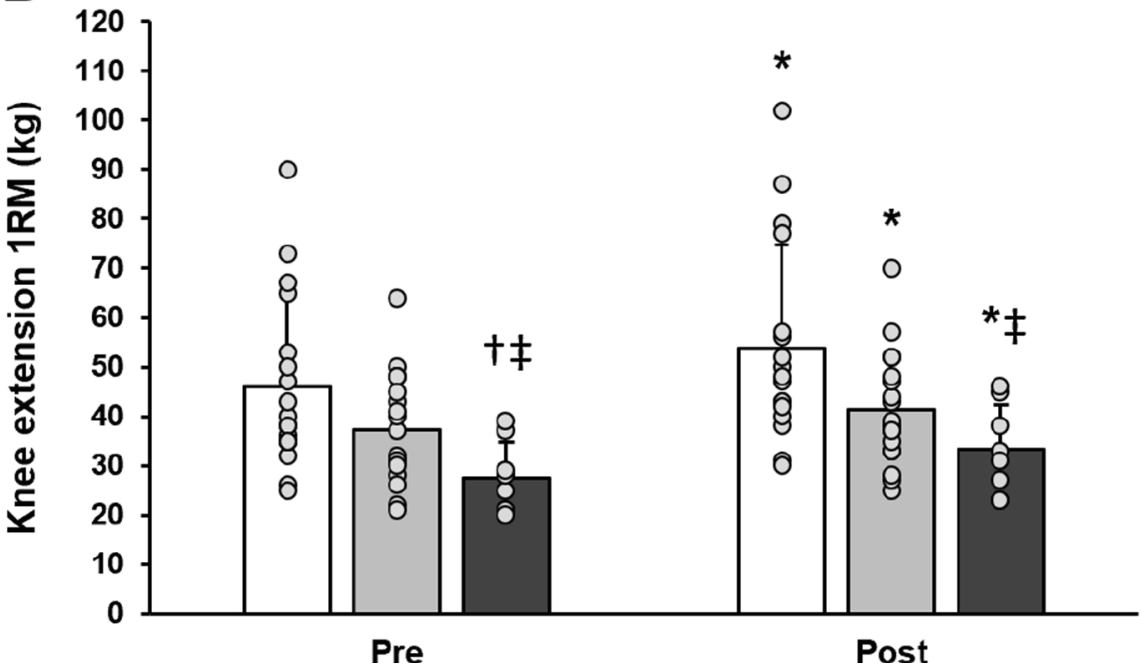

When power adaptations were analyzed at intensities relative to pre-intervention 1RM (Fig. 6), peak power was found to be improved in all groups from $30-90 \%$ 1RM $(p<0.05)$ in both KE (Fig. 6A-C) and LP (Fig. 6D-F). When compared to middle-aged adults, mobility-limited older adults and older adults had lower KE peak power both pre- and post-training from 30 to $90 \% 1 \mathrm{RM}(p<0.05)$, whereas peak power was also lower in $30 \%, 50 \%$, and $60 \% 1 \mathrm{RM}$ in the mobility-limited older adults group compared to the older adults $(p<0.05)$. In the LP exercise, mobilitylimited older adults had peak power values that were lower than those of middle-aged $(p<0.05)$ and older adults $(p<0.05)$ in all intensities analyzed. The only difference between middle-aged and older adults in the LP was found at 30\% 1RM, where although the groups were different at pre-training $(p<0.05)$, no difference was evident after the intervention $(p>0.05)$.

Functional performance

Short physical performance battery As expected per design, mobility-limited older participants had a lower SPPB score at pre-training $(7.9 \pm 0.4)$ when compared to both middle-aged $(11.9 \pm 0.1, p<0.05)$ and older adults $(11.4 \pm 0.7, p<0.05)$ (Fig. 7). Older adults also differed from middle-aged adults' scores 

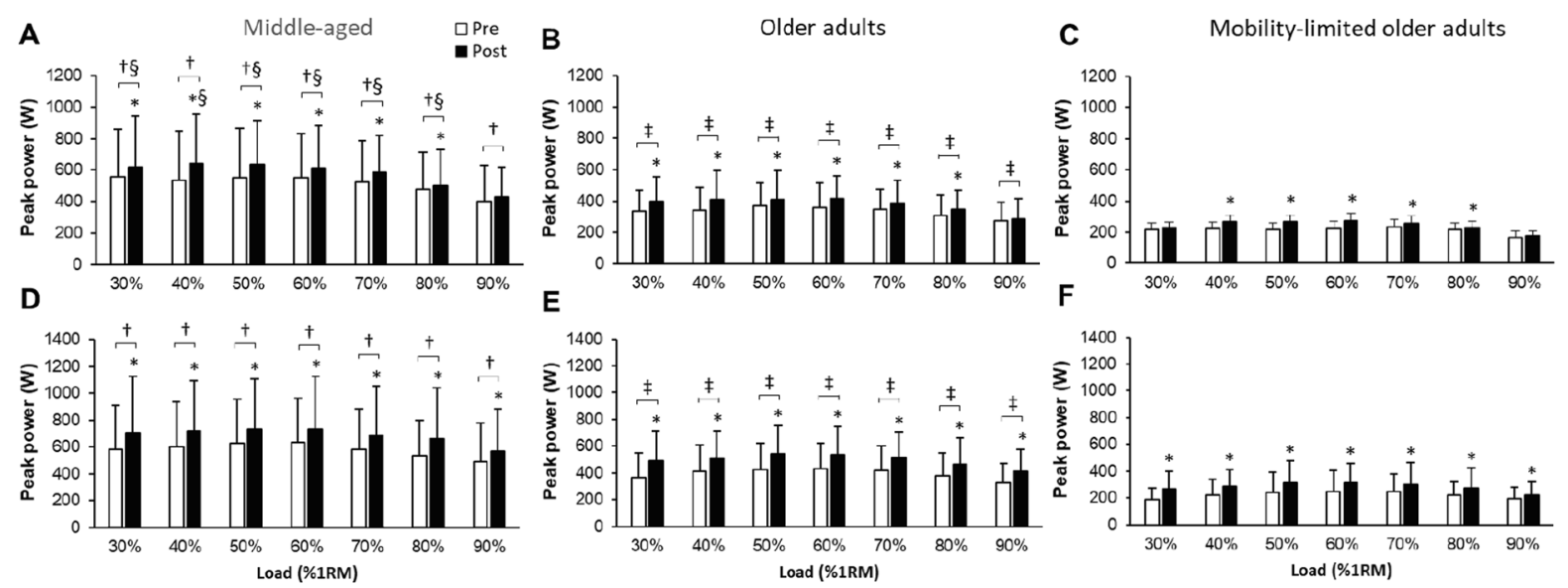

Fig. 5 Knee extension (A-C) and Leg press (D-F) peak power responses to 12 weeks of high-velocity resistance training in middle-aged $(\mathbf{A}, \mathbf{D})$, older $(\mathbf{B}, \mathbf{E})$, and mobility-limited older $(\mathbf{C}, \mathbf{F})$ participants based on 1RM at the time of testing.
$*=$ greater than pre $(p<0.05) ; \dagger=$ middle-aged adults greater than mobility-limited older adults $(p<0.05) ; \ddagger=$ older adults greater than mobility-limited older adults $(p<0.05)$; $\S=$ middle-aged adults greater than older adults $(p<0.05)$
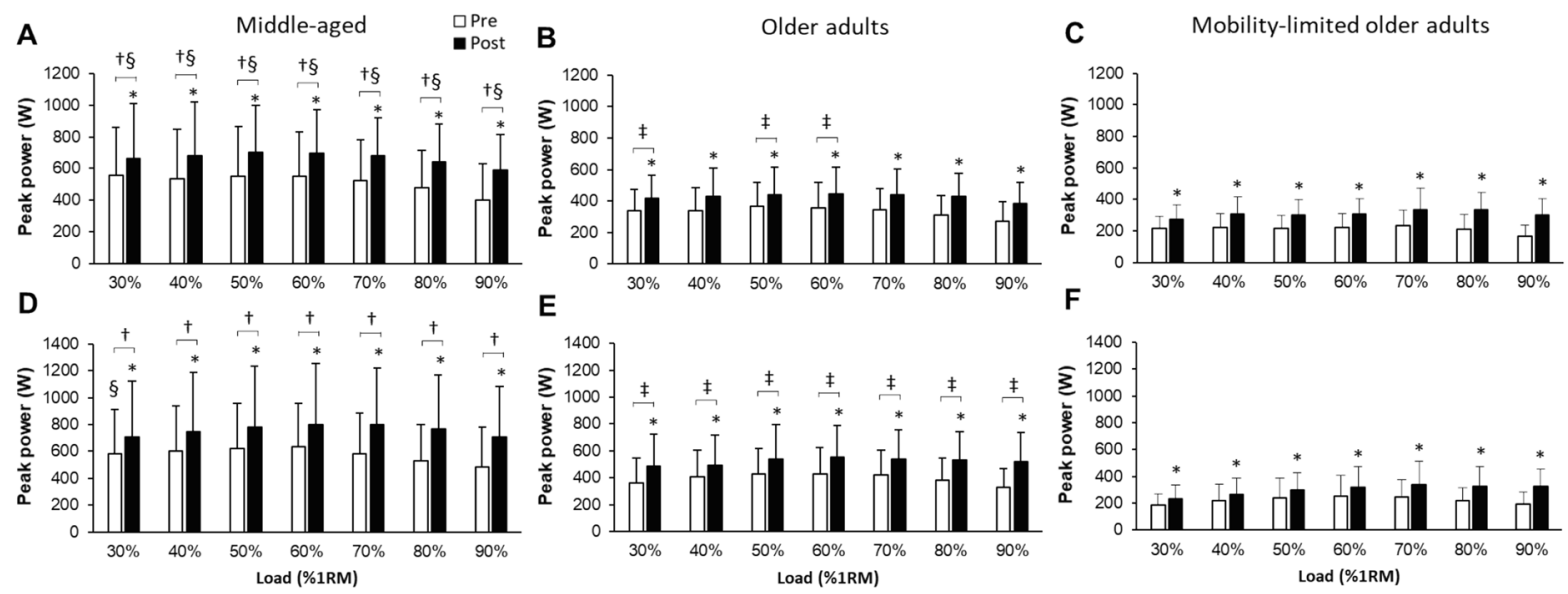

Fig. 6 Knee extension (A-C) and Leg press (D-F) peak power responses to 12 weeks of high-velocity resistance training in middle-aged (A, D), older $(\mathbf{B}, \mathbf{E})$ and mobility-limited older $(\mathbf{C}, \mathbf{F})$ participants based on pre-intervention 1RM loads.

$(p<0.05)$. After training, both mobility-limited older adults $(7.9 \pm 1.1$ vs. $10.8 \pm 1.3, p<0.05)$ and older adults $(11.4 \pm 0.7$ vs. $11.9 \pm 0.3, p<0.05)$ improved their score but not middle-aged participants $(11.9 \pm 0.1$ vs. $11.9 \pm 0.1, p>0.05)$. No difference was observed between older and middle-aged adults at post-training $(p>0.05)$, but mobility-limited older adults remained different from both the other two groups $(p<0.05)$. It should be noted that seven out
$*=$ greater than pre $(p<0.05) ; \dagger=$ middle-aged adults greater than mobility-limited older adults $(p<0.05)$; $\ddagger=$ older adults greater than mobility-limited older adults $(p<0.05)$; $\S=$ middle-aged adults greater than older adults $(p<0.05)$

of the eight mobility-limited older participants would not have been classified as mobility-limited after the intervention, whereas the remaining participant had a borderline score (i.e., 9 points).

Chair stand Prior to the intervention, middle-aged adults performed better at the 30STS test (Fig. 8A) than older adults $(18.7 \pm 3.4$ vs. $14.8 \pm 3.3$ reps, $p<0.05)$ and mobility-limited older adults $(11.3 \pm 2.1$ 


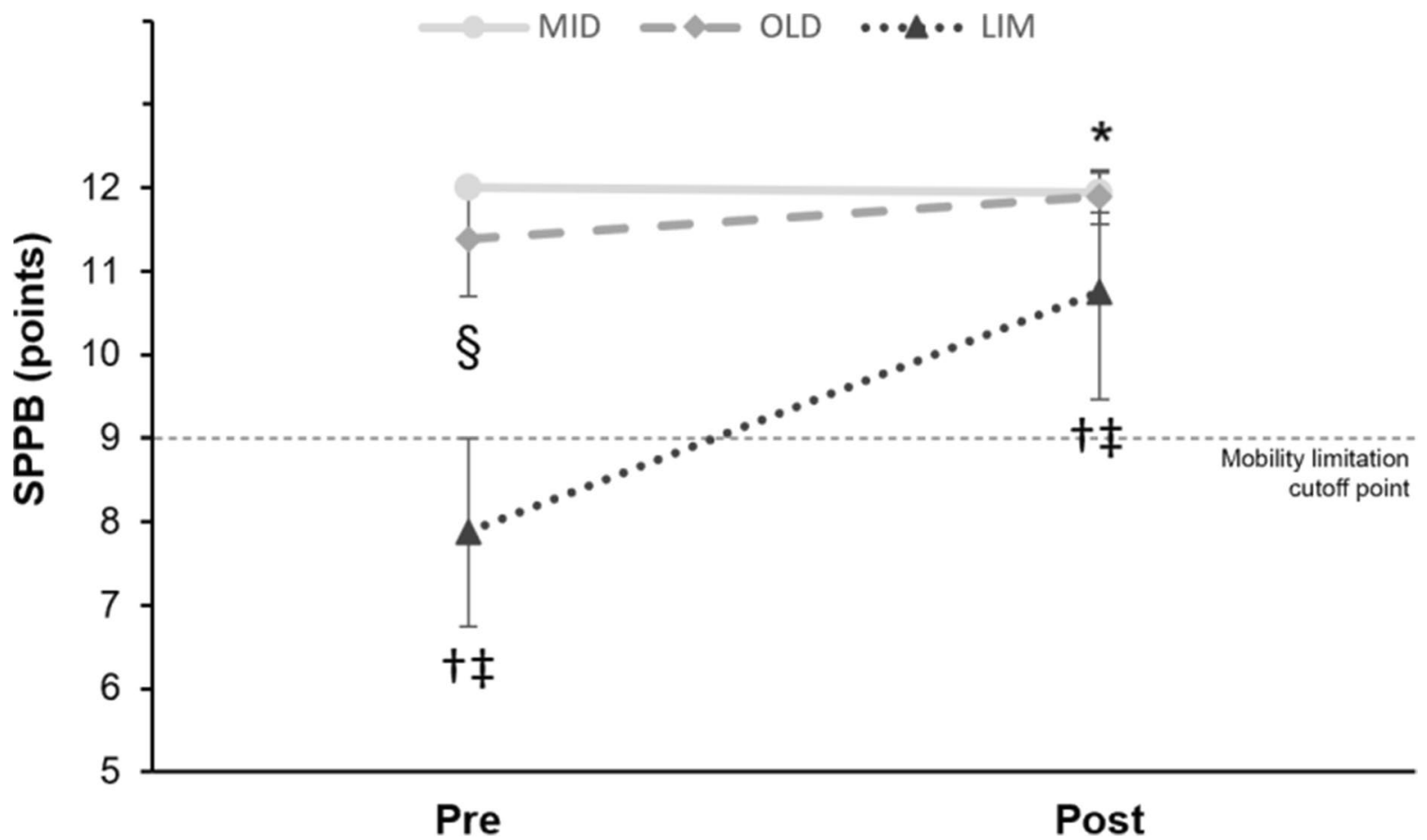

Fig. 7 Short physical performance battery (SPPB) response to 12 weeks of high-velocity resistance training in middle-aged (MID), older (OLD) and mobility-limited older (LIM) participants. SPPB total score ranges from 0 to 12 points, with higher

reps, $p<0.05)$, whilst older adults also had a better performance than those participants with mobility-limitation $(p<0.05)$. All groups improved their 30STS performance after training $(p<0.05)$, but the three groups still remained different from each other (middle-aged adults: $20.4 \pm 3.5$ reps, older adults: $15.8 \pm 3.2$ reps, mobility-limited older adults: $13.4 \pm 2.0$ reps, all $p<0.05$ ).

Stair climb Similar to the 30STS test, middle-aged adults had a better performance at the stair climb test (Fig. 8B) compared to the older adults $(4.13 \pm 0.44$ vs. $4.58 \pm 0.49 \mathrm{~s}, p<0.05)$ and mobility-limited older adults $(7.04 \pm 1.65, p<0.05)$. After training, a time point effect revealed that all three groups reduced the time necessary to perform the task $(p<0.05)$, which remained different from one another (middle-aged adults: $3.65 \pm 0.5 \mathrm{~s}$, older adults: $4.29 \pm 0.58 \mathrm{~s}$, mobility-limited older adults: $6.16 \pm 1.62$ reps, all $p<0.05$ ).

Gait speed Habitual gait speed (Fig. 8C) differed between all three groups both pre- (middle-aged: scores reflecting better function. $*=$ post greater than pre for the OLD $(p=0.005)$ and LIM $(p<0.001)$ groups; $\dagger=$ LIM lower than MID $(p<0.001)$; $\neq=$ LIM lower OLD $(p=0.032)$; $\S=$ OLD lower than MID $(p<0.001)$. Values are $($ mean \pm SD)

$1.54 \pm 0.16 \mathrm{~m} \cdot \mathrm{s}^{-1}$, older adults: $1.36 \pm 0.16 \mathrm{~m} \cdot \mathrm{s}^{-1}$, mobility-limited older adults: $1.23 \pm 0.14 \mathrm{~m} \cdot \mathrm{s}^{-1}$, $p<0.05$ ) and post-training (middle-aged adults: $1.52 \pm 0.17 \mathrm{~m} \cdot \mathrm{s}^{-1}$, older adults: $1.42 \pm 0.13 \mathrm{~m} \cdot \mathrm{s}^{-1}$, mobility-limited older adults: $1.26 \pm 0.13 \mathrm{~m} \cdot \mathrm{s}^{-1}$, $p<0.05)$, but no training effect was observed $(p>0.05)$. Maximal gait speed (Fig. 8D), on the other hand, improved in all groups after the intervention (middle-aged: $2.19 \pm 0.37$ vs. $2.26 \pm 0.24 \mathrm{~m} \cdot \mathrm{s}^{-1}$, older adults: $1.97 \pm 0.40$ vs. $2.02 \pm 0.36 \mathrm{~m} \cdot \mathrm{s}^{-1}$, mobility-limited older adults: $1.56 \pm 0.21$ vs. $1.63 \pm 0.16 \mathrm{~m} \cdot \mathrm{s}^{-1}$ ). In addition, both middle-aged and older adults were faster than the mobility-limited participants $(p<0.05)$, which were not different from one another $(p>0.05)$.

Timed up-and-go TUG performance (Fig. 8E) was also different between the groups both before (middle-aged: $5.4 \pm 0.6 \mathrm{~s}$, older adults: $6.4 \pm 0.9 \mathrm{~s}$, mobility-limited older adults: $8.9 \pm 1.9 \mathrm{~s}, \quad p<0.05)$ and after the intervention (middle-aged adults: $4.9 \pm 0.5 \mathrm{~s}$, older adults: $6.3 \pm 0.9 \mathrm{~s}$, mobility-limited older adults: 


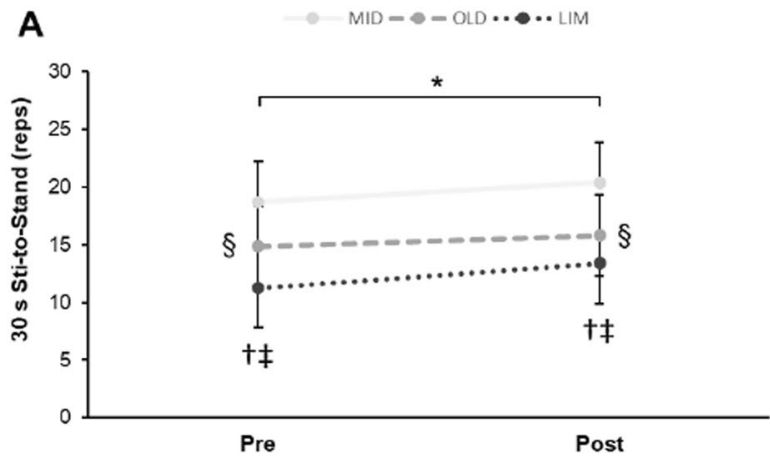

B

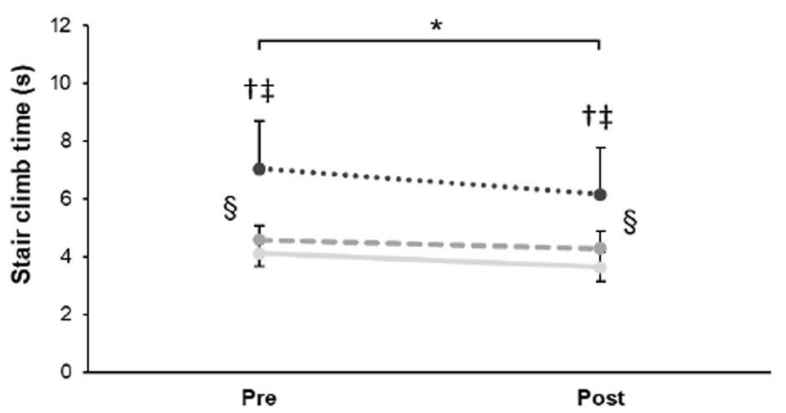

C
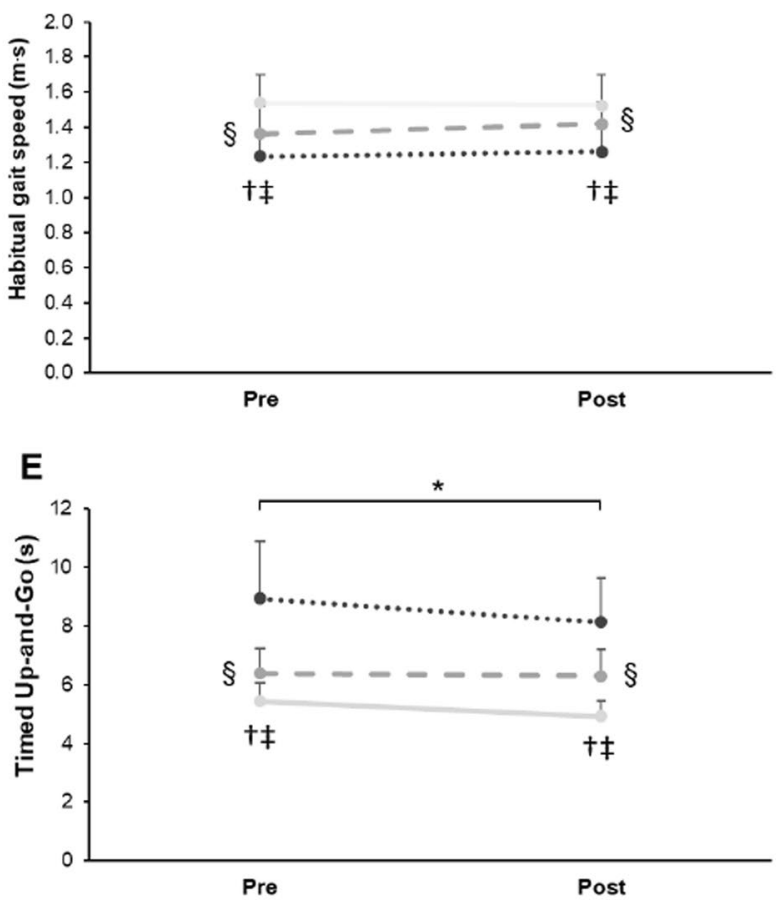

Fig. 830 s sit-to-stand (A), stair climb (B), habitual (C), and maximal (D) gait speed, as well as timed up-and-go (E) and 6-min walk (F) test responses to 12 weeks of high-velocity resistance training in middle-aged (MID), older (OLD), and

$8.1 \pm 1.5 \mathrm{~s}, p<0.05)$. A time point effect revealed that all groups improved their performance in response to training $(\mathrm{p}<0.05)$.

6-min walk test No training effect was observed for the 6-min walk test $(p>0.05)$ (Fig. 8F). Nevertheless, the test was able to differentiate the groups both pre- (middle-aged: $646.5 \pm 68.6 \mathrm{~m}$, older adults: $553.0 \pm 58.3 \mathrm{~m}$, mobility-limited older adults: $441.2 \pm 74.9 \mathrm{~m}, p<0.05)$ and post-training
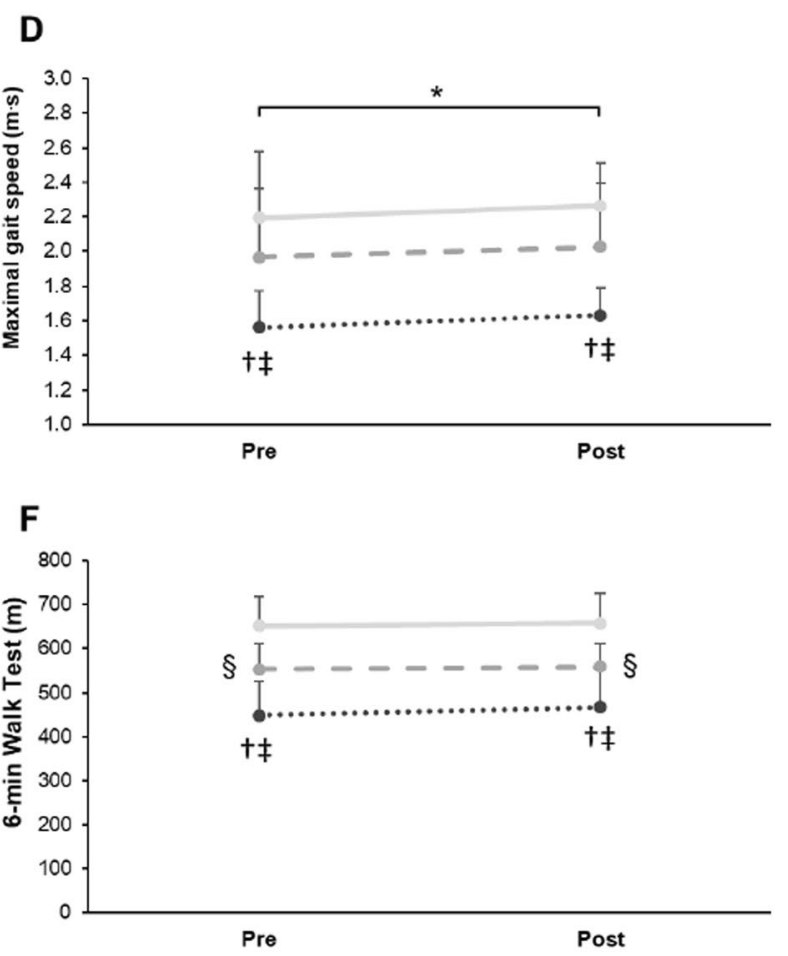

mobility-limited older (LIM) participants. $*=$ post greater than pre in all groups $(p<0.05) ; \dagger=$ LIM worse than MID (all $p<0.05$ ); $\neq=$ LIM worse OLD (all $p<0.05$ ); $\S=$ OLD worse than MID $(p<0.001)$. Values are $($ mean \pm SD $)$

(middle-aged adults: $652.8 \pm 66.4 \mathrm{~m} \cdot \mathrm{s}^{-1}$, older adults: $559.2 \pm 50.4 \mathrm{~m}$, mobility-limited older adults: $466.4 \pm 86.9 \mathrm{~m}, p<0.05)$.

Comparison with more functional, but untrained, individuals

Mobility-limited older adults vs. older adults As detailed in Supplementary Table 4, several outcomes that were found to be significantly different between 
the groups prior to the intervention were no longer different when the post-training results of the mobility-limited participants were compared to older adults pre-training values. As an example, performance in both the SPPB $(11.4 \pm 0.7$ vs. $7.9 \pm 1.1, p<0.001)$ and STS30 (14.8 \pm 3.3 vs. $11.3 \pm 2.1, p=0.010)$ tests, although better in the older adults' group pretraining, were not different post-training neither in the SPPB $(11.4 \pm 0.7$ vs. $10.8 \pm 1.3, p=0.111)$ nor STS30 $(14.8 \pm 3.3$ vs. $13.4 \pm 2.0, p=0.264)$ test. These results were also accompanied by a clear reduction in the between-group effect size for several of the neuromuscular and functional tests from large to moderate or even small.

Older adults vs. middle-aged adults When older adult's post-training results were compared to those of the middle-aged group at pre-training the majority of outcomes that were found to differ between the groups previous to the training program became non-significant (Supplementary Table 5). As an example, differences in both LP 1RM $(189.1 \pm 68.9$ vs. $145.0 \pm 40.0, \quad p=0.026)$ and quadriceps EI $(103.1 \pm 7.9$ vs. $109.4 \pm 7.0, p=0.016)$ were not observed at post-training neither for LP $(189.1 \pm 68.9$ vs. $179.2 \pm 48.5, p=0.623)$ or for quadriceps EI $(103.1 \pm 7.9$ vs. $104.6 \pm 7.0, p=0.565)$. These results were also accompanied by a clear reduction in the between-group effect size for several of the neuromuscular and functional tests from large to moderate or moderate to small.

\section{Discussion}

The main finding of the present study was that the majority of outcomes investigated improved similarly in the three groups investigated, including the functional measures in middle-aged adults. It is also noteworthy that, after just 12 weeks of HVRT, mobility-limited older adults remarkably improved their performance on the SPPB test, with most of them changing their functional classification after the intervention. These results highlight the potential of HVRT at improving neuromuscular and morphological outcomes, as well as at impacting the functional capacity of these individuals.

Performance measures are predictive of incident disability [46, 47, 57], and those individuals classified as mobility-limited are at higher risk of developing it [3, 47]. Measures to prevent functional impairments, therefore, are necessary to avoid, or at least mitigate, these risks. In the present study, a short HVRT intervention was shown to improve SPPB performance in mobility-limited older adults by almost three points on average. Considering that a one-point increase is typically considered as a clinically meaningful difference [66], the observed effect $(d=2.55)$ is substantial. More importantly, at the end of the training period just one individual was still classified as mobility-limited, whereas all the remaining participants surpassed the 9-points cut-off value (see Fig. 7).

Despite these improvements, however, the mobility-limited group still scored lower at the SPPB test when compared to the middle-aged and older adults, the latter which also improved its score after the intervention to the point that it was no longer different from the middle-aged group anymore. These results are in line with previous investigations that typically observed a more robust increase in individuals with mobility limitations [8-35\%, e.g., 29, 31, 32, 34] compared to healthy older individuals [3-7\%, 25 , 67] and underscore that a more prolonged intervention might be necessary to bring functional capacity/ mobility back to a level that is comparable/closer to that of middle-aged adults if this is indeed possible.

Improvements in SPPB were also accompanied by several positive adaptations in the other functional tests performed. These results are encouraging because these tests are typically related to distinct functional capacities, such as agility and dynamic balance (TUG) and lower body strength (30STS and SC) [66]. Although widely employed in healthy elderly individuals, the number of studies investigating HVRT functional adaptations beyond the SPPB battery in mobility-limited older adults is relatively scarce. Added to the differences in training protocols such as intensity, volume, and exercises, a comparison between the previous studies that investigated these groups separately makes it difficult to draw a conclusion related to potential differences in adaptations to individuals of different functional statuses. Here, we demonstrate that based on the same HVRT program, and contrary to what was expected, the three groups were able to comprehensively improve their functional capacity. The fact that no time point by group interaction was observed also suggests 
that these improvements occurred at the same rate between the groups.

Moreover, except for maximal gait speed, all other functional outcomes were able to differentiate between the groups, including middle-aged and older adults, suggesting that a reduction in functional capacity may already occur previous to older age, even in apparently healthy individuals. This notion is in line with several impairments already observed during the transition from middle to older age, such as reductions in muscle mass, strength, and power [13, 14], which are commonly associated with functional capacity and found to be predictors of incident disability. On the bright side, however, our results demonstrate that it is possible to build a stronger foundation in the younger individuals so that they can better deal with age-related impairments at the same time that it is possible to mitigate and even revert already installed mobility-disability in at-risk populations.

These positive functional improvements were, as expected, accompanied by both neuromuscular and morphological adaptations. Recent evidence suggests a reduction of 3 to $6 \%$ per year in muscle power output in healthy and mobility-limited older individuals $[4,6,7]$. Here, we showed that 12 weeks of HVRT resulted in 31.6 and $30.5 \%$ increase in LP peak power output when assessed based on the post-training 1RM load, and 37.9 and $43.2 \%$ when testing was repeated using the same loads as pre-training in the aforementioned groups. Thus, our results demonstrate that 3 months of HVRT were able to improve what would have been lost in 5 to 14 years due to aging and/or disuse. Cross-sectional and longitudinal data also indicate that power output is reduced as early as the fourth or fifth decade of life [e.g., 13, 69]. Middle-aged individuals in the present study increased LP peak power output by 22.5 and $34.2 \%$ on average in the same conditions mentioned above for the other groups. Accordingly, in addition to mitigating the detrimental effects of aging, HVRT also seems to provide a robust physiological reserve to middle-aged adults, likely allowing these individuals to better cope with the events related to senescence later in life.

Interestingly, the abovementioned improvements were observed throughout the entire 1RM spectrum analyzed, that is from 30-90\% 1RM, even though the training program involved only low to moderate loads. Previous data suggest that distinct functional tasks may be more related to the power output at different percentages of 1RM. As an example, gait speed appears to be more associated with power output at lower loads, whereas chair rise performance was more associated with power at higher loads [40, 70], Considering that power output is a key determinant of functional capacity [3], the improvements in peak power throughout the whole 1RM spectrum analyzed may help explain the beneficial effects observed in the different functional tasks applied. More importantly, these results greatly exceed the estimated minimal clinically important improvement and substantial improvement for LP power output in mobility-limited older adults, approximately $10 \%$ and $18 \%$, respectively, at both $40 \%$ and $70 \% 1 \mathrm{RM}$ [71],which is in agreement with the meaningful functional improvements observed.

A more detailed analysis indicates that, when KE and LP power tests were performed using post-intervention 1RM loads, improvements in all loads investigated were mostly driven or related to changes in the force component (Supplementary Figs. 7 and 8). Although no improvements were noted in the velocity component, it is important to consider that in this condition the repetitions were performed using much higher loads. Thus, the fact that all three groups were able to maintain velocities similar to pre-intervention values should be seen as an important adaptation. When post-training power tests were carried out using loads relative to the pre-intervention 1RM test, on the other hand, marked improvements were observed in the velocity component and to a lower extent in force (Supplementary Figs. 9 and 10). Since during several daily life activities such as walking, rising from a chair, or restoring balance, the external resistance imposed on the individual remains relatively constant, this latter result has an important functional and practical significance [72].

Muscle power is typically found to be more associated with functional capacity than muscle strength. Notwithstanding this, muscle strength is a wellknown independent predictor of mortality and disability and is especially important for weight-bearing activities. After the intervention, all training groups improved LP and KE 1RM. The capacity of middleaged and healthy older adults to respond to resistance training containing a power component has already been established for quite some time [73, 74]. However, in these classic studies, only a portion of the training volume was actually performed explosively 
( 25\%), whereas the rest was completed using workloads and movement velocities typically employed in traditional resistance training. Here, we demonstrate that even middle-aged individuals are able to improve considerably their maximal dynamic strength while using only low to moderate loads performed as fast as possible, at least in the short term. Substantial improvements were also found in the mobility-limited older group, in line with previous investigations [29, $32,35,43,75-77]$. Of note, although mobility-limited older participants still had lower KE 1RM values compared to middle-aged adults after the intervention, the former was not found to be different from older adults post-training. This highlights the capacity of HVRT to recover a healthier phenotype in these individuals.

Several age-related changes in the nervous system may compromise the ability of older individuals to fully drive the motor pool [36, 78]. Although some data suggest that peak sEMG amplitude is relatively well preserved during maximal isometric contractions [78], it has previously been demonstrated that neuromuscular activation is impaired in some older adults, especially those with limited mobility [79] and that this may contribute to age-related weakness. Our results partially agree with this notion by showing an impaired VL activation and MVIC force in mobilitylimited individuals compared to middle-aged adults. More importantly, we were able to show a training effect on both VL and RF sEMG amplitude for all the groups investigated, which were accompanied by improvements in MVIC for middle-aged and mobility-limited participants, but not the well-functioning older adults. Considering the significant improvements observed on maximal dynamic strength and power output for all the three groups, testing specificity may at least partially explain the lack of effect for the older adults [78, 79].

With respect to antagonist coactivation, BF sEMG was found to be lower in mobility-limited older adults than in the other two groups. At first, this result is counterintuitive, as it would be expected to be higher in the former. However, it should be noted that $\mathrm{BF}$ results were reported in absolute values, as we were unable to normalize the sEMG amplitude to that of a knee flexion maximal isometric contraction. Although the lack of normalization might hinder comparison between the groups, within-group comparison does not suggest a training effect on antagonist coactivation and, thus, training-related adaptations in muscle strength, power, and function were likely not related to changes in coactivation. This notion is in line with cross-sectional data indicating that changes in coactivation were not responsible for deficits in dynamic force production when comparing middle-aged and older adults with and without limitations [36].

Ultrasound-derived measures have been frequently used to investigate characteristics of skeletal muscle morphology [80]. In the present study, we showed an increase in quadriceps muscles thickness in the three groups investigated, suggesting that all groups were able to improve muscle size in response to the intervention. Percent changes and effect sizes, however, indicate that the hypertrophic ability of mobility-limited older adults' muscle might be reduced in comparison to the other groups. Notwithstanding this, our results are in line with those of Radaelli et al. [28] and Nogueira et al. [81], who also showed an increase in quadriceps muscle thickness in response to 12 and 10 weeks of HVRT in healthy older men and women. Muscle quality, on the other hand, only improved in middle-aged and older adults. This might be related to, or be suggestive of, an impaired intramuscular environment or remodeling capacity in mobilitylimited individuals. Higher echo intensity values are believed to be reflective of greater intramuscular adiposity and fibrous tissue content $[82,83]$, both of which may hinder muscle function and metabolism [80]. Another possibility is that periods longer than 12 weeks of training may be necessary for improvements to be evident in this population, which is supported by the small, but significant increases in muscle quality noted in some of the individual muscles (Supplemental Fig. 3).

Remarkably, no difference in quadriceps muscle thickness was observed between older individuals with and without mobility limitations. Previous longitudinal data evidenced that only mobility-limited older adults, but not healthy older adults, showed reductions in muscle mass after a 3-year followup period and, accordingly, muscle thickness was expected to be reduced in those with mobility limitations [7]. Considering the large differences in functional capacity and power observed previous to the intervention between the groups, our results seem to advocate the notion that altered neuromuscular function is the critical early determinant of muscle power 
loss with aging, as suggested by Reid et al. [7]. In addition, it has recently been shown that RF and VL cross-sectional area and echo intensity values were not predictive of fast gait speed in older individuals, which led the authors to suggest a potential dissociation between skeletal muscle morphology and gait speed [84]. The absence of an effect on quadriceps echo intensity, coupled with marked functional performance improvements, is suggestive that improvements in skeletal muscle morphology markers may not be a pre-requisite to improve functional capacity in the short term, at least in the mobility-limited older adults. Nevertheless, skeletal muscle has important endocrine, metabolic and signaling functions [e.g., $85,86]$, and the mechanisms behind the fact that mobility-limited older adults were able to improve muscle size, but not quality deserves further attention.

Our results are reinforced by the control period used, which confirms the overall stability of our measures independently of the training group investigated and demonstrates that our familiarization procedures were adequately employed prior to the intervention. More important, these results support the conclusion that improvements observed after the intervention period were indeed a direct result of the exercise regimen employed. In addition, the intervention was effective with few adverse events directly related to training reported. Only three of the events definitely or possibly related to training were judged as moderate, whereas the remaining eight events were judged as mild. Furthermore, practically none of the events reported resulted in changes and/or absences to the training program (i.e., 950 of 952 administered sessions were completed). Considering that most HVRT investigations do not fully report on adverse events, our data can assist in understanding aspects related to the safety and implementation of HVRT interventions.

\section{Limitations}

Potential limitations should be considered when interpreting the results of the current investigation. First, caution is needed when extrapolating our results to severely limited individuals, as our sample included only individuals with mild to moderate limitations in mobility. The isometric testing condition might also have limited our capacity to observe potential training effects in both agonist and antagonist neuromuscular activation, as agonist activation amplitude deficits seem to be more evident at higher movement velocities [36]. In addition, sEMG amplitude data were not corrected to account for the influence of subcutaneous adipose tissue, even though no difference was previously found between original and corrected sEMG values when comparing middle-aged and older adults with and without mobility-limitation [36]. Another possible limitation is the order of the loads used during the power tests, which was not randomized for logistic reasons. Although unlikely, performance on the final loads might have been influenced by the previous ones. Previous data also suggests that nutritional status may influence muscle response to exercise, and the presence of a dietary control may have provided a greater insight into the morphological results observed, especially in the mobility-limited participants [87]. Notwithstanding this, participants were appropriately instructed to maintain their eating habits throughout the duration of the study. Finally, although we were unable to reach the estimated sample size for the mobility-limited older adults' group, the statistical model adopted (i.e., GEE) seemed capable of differentiating this group from the others (e.g., Fig. 8), while also highlighting the presence (or lack thereof) of training-related effects when it differed from the other groups (e.g., Fig. 3). We also provided a comprehensive report of both within- and between-group effect sizes (Suppl. Tables 2 and 3), which should allow readers to have a more informed understanding of our findings.

\section{Conclusions}

Overall, our results demonstrate that a HVRT intervention can build a stronger foundation in middleaged individuals so that they can better deal with age-related impairments at the same time that it can mitigate already present physiological and functional impairments in older adults with and without mobility-limitation.

Acknowledgements We would like to thank all subjects who took part in the study for their genuine effort. We would also like to acknowledge L. Nanini, M. Borba, S. Nickel, T. Castro, M. Fonseca, L. Linhares, C. Zillig, L. Cavalli, B. Veiga, G. Schiller, and B. Ornelas for their assistance in the training sessions. 
Funding This study was financed in part by the Fundação de Amparo à Pesquisa do estado do Rio Grande do Sul (FAPERGS), Brazil (19/2551-0001979-3).

Data availability Data from the present study will be made available upon request to the corresponding author.

\section{Declarations}

Conflict of interest The authors declare no competing interests.

\section{References}

1. Instituto Brasileiro de Geografia e Estatística (IBGE). Projeção da população do Brasil por sexo e idade para o período 2000-2060. Rio de Janeiro: IBGE, 2013.

2. World Health Organization (WHO). World report on ageing and health. Geneva: WHO. 2015. https://apps.who.int/ iris/handle/10665/186463. Accessed 26 Jan 2022

3. Reid KF, Fielding R. a. Skeletal Muscle Power: A Critical Determinant of Physical Functioning in Older Adults. Exerc Sport Sci Rev. 2012;40:4-12.

4. Clark DJ, Pojednic RM, Reid KF, Patten C, Pasha EP, Phillips EM, et al. Longitudinal decline of neuromuscular activation and power in healthy older adults. J Gerontol Ser A Biol Sci Med Sci. 2013;68:1419-25.

5. Metter EJ, Conwit R, Tobin J, Fozard JL. Age-associated loss of power and strength in the upper extremities in women and men. J Gerontol - Ser A Biol Sci Med Sci. 1997;52:267-76.

6. Mitchell WK, Williams J, Atherton P, Larvin M, Lund J, Narici M. Sarcopenia, dynapenia, and the impact of advancing age on human skeletal muscle size and strength; a quantitative review. Front Physiol. 2012;3:1-18.

7. Reid KF, Pasha E, Doros G, Clark DJ, Patten C, Phillips $\mathrm{EM}$, et al. Longitudinal decline of lower extremity muscle power in healthy and mobility-limited older adults: Influence of muscle mass, strength, composition, neuromuscular activation and single fiber contractile properties. Eur J Appl Physiol. 2014;114:29-39.

8. Skelton DA, Greig CA, Davies JM, Young A. Strength, power and related functional ability of healthy people aged 65-89 years. Age Ageing. 1994;23:371-7.

9. Carabello RJ, Reid KF, Clark DJ, Phillips EM, Fielding RA. Lower extremity strength and power asymmetry assessment in healthy and mobility-limited populations: reliability and association with physical functioning. Clin Exp Res. 2010;22:324-9.

10. Foldvari M, Clark M, Laviolette LC, Bernstein MA, Kaliton D, Castaneda C, et al. Association of muscle power with functional status in community-dwelling elderly women. J Gerontol Med Sci. 2000;55A:M192-9.

11. Jacob ME, Travison TG, Ward RE, Latham NK, Leveille SG, Jette AM, et al. Neuromuscular attributes associated with lower extremity mobility among community-dwelling older adults. J Gerontol Ser A. 2018;74:544-9.

12. Suzuki T, Bean JF, Fielding RA. Muscle power of the ankle flexors predicts functinal performance in community-dwelling older women. J Am Geriatr Soc. 2001;49:1161-7.

13. Leyva A, Balachandran A, Signorile JF. Lower-body torque and power declines across six decades in three hundred fifty-seven men and women: a cross-sectional study with normative values. J Strength Cond Res. 2016;30:141-58.

14. Roberts BM, Lavin KM, Many GM, Thalacker-Mercer A, Merritt EK, Bickel CS, et al. Human neuromuscular aging sex differences revealed at the myocellular level. Exp Gerontol Elsevier Inc. 2018;106:116-24.

15. ACSM. American College of Sports Medicine. Exercise and physical activity for older adults. Med Sci Sports Exerc. 2009;41:1510-30.

16. Byrne C, Faure C, Keene DJ, Lamb SE. Ageing, muscle power and physical function: a systematic review and implications for pragmatic training interventions. Sports Med New Zealand. 2016;46:1311-32.

17. Lavin KM, Roberts BM, Fry CS, Moro T, Rasmussen $\mathrm{BB}$, Bamman MM. The importance of resistance exercise training to combat neuromuscular aging. Physiology. 2019;34:112-22.

18. Peterson MD, Rhea MR, Sen A, Gordon PM. Resistance exercise for muscular strength in older adults: a metaanalysis. Ageing Res Rev. 2010;9:226-37.

19. Peterson MD, Sen A, Gordon PM. Influence of resistance exercise on lean body mass in aging adults: a metaanalysis. Med Sci Sports Exerc. 2011;43:249-58.

20. Straight CR, Lindheimer JB, Brady AO, Dishman RK, Evans EM. Effects of resistance training on lowerextremity muscle power in middle-aged and older adults: a systematic review and meta-analysis of randomized controlled trials. Sports Med New Zealand. 2016;46:353-64.

21. Tschopp M, Sattelmayer MK, Hilfiker R. Is power training or conventional resistance training better for function in elderly persons? A meta-analysis Age Ageing England. 2011;40:549-56.

22. Bottaro M, Machado SN, Nogueira W, Scales R, Veloso J. Effect of high versus low-velocity resistance training on muscular fitness and functional performance in older men. Eur J Appl Physiol Germany. 2007;99:257-64.

23. Stec MJ, Thalacker-Mercer A, Mayhew DL, Kelly NA, Tuggle CS, Merritt EK, et al. Randomized, four-arm, dose-response clinical trial to optimize resistance exercise training for older adults with age-related muscle atrophy. Exp Gerontol Elsevier. 2017;99:98-109.

24. Surakka J, Aunola S, Nordblad T, Karppi S-L, Alanen E. Feasibility of power-type strength training for middle aged men and women: self perception, musculoskeletal symptoms, and injury rates. Br J Sports Medicine. 2003;37:131-6.

25. Earles DR, Judge JO, Gunnarsson OT. Velocity training induces power-specific adaptations in highly functioning older adults. Arch Phys Med Rehabil USA. 2001;82:872-8.

26. Balachandran AT, Gandia K, Jacobs KA, Streiner DL, Eltoukhy M, Signorile JF. Power training using pneumatic machines vs plate-loaded machines to improve muscle power in older adults. Exp Gerontol England. 2017;98:134-42. 
27. Henwood TR, Riek S, Taaffe DR. Strength versus muscle power-specific resistance training in communitydwelling older adults. J Gerontol A Biol Sci Med Sci USA. 2008;63:83-91.

28. Radaelli R, Brusco CM, Lopez P, Rech A, Machado CLF, Grazioli R, et al. Higher muscle power training volume is not determinant for the magnitude of neuromuscular improvements in elderly women. Exp Gerontol England. 2018;110:15-22.

29. Balachandran A, Krawczyk SN, Potiaumpai M, Signorile JF. High-speed circuit training vs hypertrophy training to improve physical function in sarcopenic obese adults: a randomized controlled trial. Exp Gerontol England. 2014;60:64-71.

30. Cadore EL, Casas-Herrero A, Zambom-Ferraresi F, Idoate F, Millor N, Gomez M, et al. Multicomponent exercises including muscle power training enhance muscle mass power output and functional outcomes in institutionalized frail nonagenarians Age (Dordr). Netherlands. 2014;36:773-85.

31. Bean JF, Herman S, Kiely DK, Frey IC, Leveille SG, Fielding RA, et al. Increased velocity exercise specific to task (InVEST) training: a pilot study exploring effects on leg power, balance, and mobility in community-dwelling older women. J Am Geriatr Soc. 2004;52:799-804.

32. Bean JF, Kiely DK, LaRose S, O’Neill E, Goldstein R, Frontera WR. Increased velocity exercise specific to task training versus the national institute on aging's strength training program: changes in limb power and mobility. J Gerontol - Ser A Biol Sci Med Sci. 2009;64:983-91.

33. Reid KF, Callahan DM, Carabello RJ, Phillips EM, Frontera WR, Fielding RA. Lower extremity power training in elderly subjects with mobility limitations: a randomized controlled trial. Aging Clin Exp Res Germany. 2008;20:337-43.

34. Reid KF, Martin KI, Doros G, Clark DJ, Hau C, Patten $\mathrm{C}$, et al. Comparative effects of light or heavy resistance power training for improving lower extremity power and physical performance in mobility-limited older adults. J Gerontol A Biol Sci Med Sci USA. 2015;70:374-80.

35. Hvid LG, Strotmeyer ES, Skjodt M, Magnussen LV, Andersen M, Caserotti P. Voluntary muscle activation improves with power training and is associated with changes in gait speed in mobility-limited older adults a randomized controlled trial. Exp Gerontol England. 2016;80:51-6.

36. Clark DJ, Patten C, Reid KF, Carabello RJ, Phillips EM, Fielding RA. Impaired voluntary neuromuscular activation limits muscle power in mobility-limited older adults. $\mathrm{J}$ Gerontol - Ser A Biol Sci Med Sci. 2010;65(A):495-502.

37. Pojednic RM, Clark DJ, Patten C, Reid K, Phillips EM, Fielding RA. The specific contributions of force and velocity to muscle power in older adults. Exp Gerontol Elsevier BV. 2012;47:608-13.

38. de Vos NJ, Singh NA, Ross DA, Stavrinos TM, Orr R, Fiatarone Singh MA. Optimal load for increasing muscle power during explosive resistance training in older adults. J Gerontol A Biol Sci Med Sci USA. 2005;60:638-47.

39. Katsoulis K, Stathokostas L, Amara CE. The effects of high versus low-intensity power training on muscle power outcomes in healthy, older adults: a systematic review. J Aging Phys Act USA. 2019;27:422-39.

40. Cuoco A, Callahan DM, Sayers S, Frontera WR, Bean J, Fielding RA. Impact of muscle power and force on gait speed in disabled older men and women. J Gerontol - Ser A Biol Sci Med Sci. 2004;59:1200-6.

41. Alcazar J, Rodriguez-Lopez C, Ara I, Alfaro-Acha A, Rodriguez-Gomez I, Navarro-Cruz R, et al. Force-velocity profiling in older adults: An adequate tool for the management of functional trajectories with aging. Exp Gerontol England. 2018;108:1-6.

42. Marsh AP, Miller ME, Rejeski WJ, Hutton SL, Kritchevsky SB. Lower extremity muscle function after strength or power training in older adults. J Aging Phys Act USA. 2009; 17:416-43.

43. Reid KF, Martin KI, Doros G, Clark DJ, Hau C, Patten $\mathrm{C}$, et al. Comparative effects of light or heavy resistance power training for improving lower extremity power and physical performance in mobility-limited older adults. J Gerontol A Biol Sci Med Sci. 2015;70:374-80.

44. Webber SC, Porter MM. Effects of ankle power training on movement time in mobility-impaired older women. Med Sci Sports Exerc USA. 2010;42:1233-40.

45. Fielding RA, LeBrasseur NK, Cuoco A, Bean J, Mizer K, Fiatarone Singh MA. High-velocity resistance training increases skeletal muscle peak power in older women. J Am Geriatr Soc USA. 2002;50:655-62.

46. Guralnik JM, Simonsick EM, Ferrucci L, Glynn RJ, Berkman LF, Blazer DG, et al. A short physical performance battery assessing lower extremity function: association with self-reported disability and prediction of mortality and nursing home admission. J Gerontol Med Sci. 1994;49:M85-94.

47. Guralnik JM, Ferrucci L, Simonsick EM, Salive ME, Wallace RB. Lower-extremity function in persons over the age of 70 years as a predictor of subsequent disability. N Engl J Med. 1995;332:556-62.

48. Lopez P, Pinto MD, Pinto RS. Does rest time before ultrasonography imaging affect quadriceps femoris muscle thickness, cross-sectional area and echo intensity measurements? Ultrasound Med Biol. 2019;45:612-6.

49. Kumagai K, Abe T, Brechue WF, Ryushi T, Takano S, Mizuno M. Sprint performance is related with muscle fascicle length in male $100 \mathrm{~m}$-sprinters. J Appl Physiol. 2000;88:811-6.

50. Korhonen MT, Mero AA, Alén M, Sipilä S, Häkkinen K, Liikavainio $\mathrm{T}$, et al. Biomechanical and skeletal muscle determinants of maximum running speed with aging. Med Sci Sport Exerc. 2009; 41:844-56.

51. Schaun GZ, Pinto SS, Brasil B, Nunes GN, Alberton CL. Neuromuscular adaptations to sixteen weeks of wholebody high-intensity interval training compared to ergometer-based interval and continuous training. J Sport Sci. 2019;37(14):1561-9.

52. Andrade LS, Pinto SS, Silva MR, Schaun GZ, Portella EG, Nunes GN, et al. Water-based continuous and interval training in older women cardiorespiratory and neuromuscular outcomes (WATER study). Exp Gerontol Elsevier Inc. 2020;134:110914.

53. Hermens HJ, Freriks B, Disselhorst-Klug C, Rau G. Development of recommendations for SEMG sensors and 
sensor placement procedures. J Electromyogr Kinesiol. 2000;10:361-74.

54. Ni M, Signorile JF. High-speed resistance training modifies load-velocity and load-power relationships in parkinson's disease. $\mathrm{J}$ strength Cond Res USA. 2017;31:2866-75.

55. Sayers SP, Gibson K. Effects of high-speed power training on muscle performance and braking speed in older adults. J Aging Res USA. 2012;2012:426278.

56. Vasunilashorn S, Coppin AK, Patel KV, Lauretani F, Ferrucci $\mathrm{L}$, Bandinelli $\mathrm{S}$, et al. Use of the short physical performance battery score to predict loss of ability to walk 400 meters: analysis from the InCHIANTI study. J Gerontol - Ser A Biol Sci Med Sci. 2009;64:223-9.

57. Guralnik JM, Ferrucci L, Pieper CF, Leveille SG, Markides KS, Ostir GV, et al. Lower extremity function and subsequent disability: consistency across studies, predictive models, and value of gait speed alone compared with the short physical performance battery. J Gerontol Ser A Biol Sci Med Sci. 2000;55:M221-31.

58. Studenski S, Faulkner K, Inzitari M, Brach J, Chandler J, Cawthon $\mathrm{P}$, et al. Gait speed and survival in older adults. JAMA J Am Med Assoc. 2011;305:50-8.

59. Yates T, Zaccardi F, Dhalwani NN, Davies MJ, Bakrania $\mathrm{K}$, Celis-Morales CA, et al. Association of walking pace and handgrip strength with all-cause, cardiovascular, and cancer mortality: a UK Biobank observational study. Eur Heart J. 2017;38:3232-40.

60. Holviala JHS, Sallinen JM, Kraemer WJ, Alen M, Hakkinen K. Effects of strength training on muscle strength characteristics, functional capabilities, and balance in middle-aged and older women. Strength Cond. 2006;20:336-44.

61. Pereira A, Izquierdo M, Silva AJ, Costa AM, Bastos E, Gonzalez-Badillo JJ, et al. Effects of high-speed power training on functional capacity and muscle performance in older women. Exp Gerontol England. 2012;47:250-5.

62. Bean JF, Kiely DK, Herman S, Leveille SG, Mizer K, Frontera WR, et al. The relationship between leg power and physical performance in mobility-limited older people. J Am Geriatr Soc. 2002;50:461-7.

63. Bohannon RW. Reference values for the timed up and go test: a descriptive meta-analysis. J Geriatr Phys Ther. 2006;29:64-8.

64. Brzycki M. Strength testing-predicting a one-rep max from reps-to-fatigue. J Phys Educ Recreat Danc. 1993;64:88-90.

65. Richardson DL, Duncan MJ, Jimenez A, Juris PM, Clarke ND. Effects of movement velocity and training frequency of resistance exercise on functional performance in older adults: a randomised controlled trial. Eur J Sport Sci England. 2019;2:234-46.

66. Perera S, Mody SH, Woodman RC, Studenski SA. Meaningful change and responsiveness in common physical performance measures in older adults. J Am Geriatr Soc USA. 2006;54:743-9.

67. Englund DA, Sharp RL, Selsby JT, Ganesan SS, Franke WD. Resistance training performed at distinct angular velocities elicits velocity-specific alterations in muscle strength and mobility status in older adults. Exp Gerontol England. 2017;91:51-6.
68. Rikli RE, Jones CJ. Senior fitness test manual. 2nd ed. Champaign, IL: Human Kinetics; 2013.

69. Kennis E, Verschueren S, Van Roie E, Thomis M, Lefevre J, Delecluse C. Longitudinal impact of aging on muscle quality in middle-aged men. Age (Omaha). 2014;36:9689.

70. Puthoff ML, Nielsen DH. Relationships among impairmeets in lower-extremity strength and power, functional limitations, and disability in older adults. Phys Ther. 2007;87:1334-47.

71. Kirn DR, Reid KF, Hau C, Phillips EM, Fielding RA. What is a clinically meaningful improvement in legextensor power for mobility-limited older adults? J Gerontol A Biol Sci Med Sci USA. 2016;71:632-6.

72. Sayers SP, Gibson K. A comparison of high-speed power training and traditional slow-speed resistance training in older men and women. J strength Cond Res USA. 2010;24:3369-80.

73. Izquierdo M, Häkkinen K, Ibañez J, Garrues M, Antón A, Zúñiga A, et al. Effects of strength training on muscle power and serum hormones in middle-aged and older men. J Appl Physiol. 2001;90:1497-507.

74. Newton RU, Hakkinen K, Hakkinen A, McCormick M, Volek JS, Kraemer WJ. Mixed-methods resistance training increases power and strength of young and older men. Med Sci Sports Exerc. 2002;34:1367-75.

75. Miszko TA, Cress ME, Slade JM, Covey CJ, Agrawal SK, Doerr CE. Effect of strength and power training on physical function in community-dwelling older adults. J Gerontol A Biol Sci Med Sci USA. 2003;58:171-5.

76. Bean JF, Leveille SG, Kiely DK, Bandinelli S, Guralnik JM, Ferrucci L. A Comparison of leg power and leg strength within the InCHIANTI study: which influences mobility more? J Gerontol Ser A Biol Sci Med Sci. 2003;58:M728-33.

77. Reid KF, Callahan DM, Carabello RJ, Phillips EM, Frontera WR, Fielding RA. Lower extremity power training in elderly subjects with mobility limitations: a randomized controlled trial. Aging Clin Exp Res. 2008;20:337-43.

78. Klass M, Baudry S, Duchateau J. Voluntary activation during maximal contraction with advancing age: a brief review. Eur J Appl Physiol Germany. 2007;100:543-51.

79. Clark DJ, Patten C, Reid KF, Carabello RJ, Phillips EM, Fielding RA. Muscle performance and physical function are associated with voluntary rate of neuromuscular activation in older adults. J Gerontol - Ser A Biol Sci Med Sci. 2011;66:115-21.

80. Correa-de-Araujo R, Harris-Love MO, Miljkovic I, Fragala MS, Anthony BW, Manini TM. The need for standardized assessment of muscle quality in skeletal muscle function deficit and other aging-related muscle dysfunctions: a symposium report. Front Physiol. 2017;8:1-19.

81. Nogueira W, Gentil P, Mello SNM, Oliveira RJ, Bezerra AJC, Bottaro M. Effects of power training on muscle thickness of older men. Int J Sports Med Germany. 2009;30:200-4.

82. Reimers K, Reimers CD, Wagner S, Paetzke I, Pongratz DE. Skeletal muscle sonography: a correlative study of echogenicity and morphology. J Ultrasound Med. 1993;12:73-7. 
83. Young H-J, Jenkins NT, Zhao Q, Mccully KK. Measurement of intramuscular fat by muscle echo intensity. Muscle Nerve John Wiley \& Sons Ltd. 2015;52:963-71.

84. Komforti D, Joffe C, Magras A, Peller A, Garbe E, Garib $\mathrm{R}$, et al. Does skeletal muscle morphology or functional performance better explain variance in fast gait speed in older adults? Aging Clin Exp Res. 2021;33:921-31.

85. Pedersen BK. Muscle as a secretory organ. Compr Physiol. 2013;3:1337-62.

86. Vechetti IJ Jr, Valentino T, Mobley CB, McCarthy JJ. The role of extracellular vesicles in skeletal muscle and systematic adaptation to exercise. J Physiol John Wiley \& Sons Ltd. 2021;599:845-61.
87. Fiatarone MA, O'Neill EF, Ryan ND, Clements KM, Solares GR, Nelson ME, et al. Exercise training and nutritional supplementation for physical frailty in very elderly people. N Engl J Med Massachusetts Medical Society. 1994;330:1769-75.

Publisher's note Springer Nature remains neutral with regard to jurisdictional claims in published maps and institutional affiliations. 